\title{
A Measurement of the Charm and Bottom Forward-Backward Asymmetries using D Mesons at LEP
}

\author{
OPAL COLLABORATION
}

\begin{abstract}
A measurement of the charm and bottom forward-backward asymmetry in $\mathrm{e}^{+} \mathrm{e}^{-}$annihilations is presented at energies on and around the peak of the $\mathrm{Z}^{0}$ resonance. Decays of the $\mathrm{Z}^{0}$ into charm and bottom quarks are tagged using $\mathrm{D}$ mesons identified in about 4 million hadronic decays of the $\mathrm{Z}^{0}$ boson recorded with the OPAL detector at LEP between 1990 and 1995. Approximately $33000 \mathrm{D}$ mesons are tagged in seven different decay modes. From these the charm and bottom asymmetries are measured in three energy ranges around the $\mathrm{Z}^{0}$ peak:

$$
\begin{array}{lll}
A_{\mathrm{FB}}^{\mathrm{c}}=0.039 \pm 0.051 \pm 0.009 & A_{\mathrm{FB}}^{\mathrm{b}}=-0.086 \pm 0.108 \pm 0.029 & \left\langle E_{c m}\right\rangle=89.45 \mathrm{GeV} \\
A_{\mathrm{FB}}^{\mathrm{c}}=0.063 \pm 0.012 \pm 0.006 & A_{\mathrm{FB}}^{\mathrm{b}}=0.094 \pm 0.027 \pm 0.022 & \left\langle E_{c m}\right\rangle=91.22 \mathrm{GeV} \\
A_{\mathrm{FB}}^{\mathrm{c}}=0.158 \pm 0.041 \pm 0.011 & A_{\mathrm{FB}}^{\mathrm{b}}=-0.021 \pm 0.090 \pm 0.026 & \left\langle E_{c m}\right\rangle=93.00 \mathrm{GeV}
\end{array}
$$
\end{abstract}

The results are in agreement with the predictions of the standard model and other measurements at LEP. 


\section{The OPAL Collaboration}

G. Alexander ${ }^{23}$, J. Allison ${ }^{16}$, N. Altekamp ${ }^{5}$, K. Ametewee ${ }^{25}$, K.J. Anderson ${ }^{9}$, S. Anderson ${ }^{12}$, S. Arcelli ${ }^{2}$, S. Asai ${ }^{24}$, D. Axen ${ }^{29}$, G. Azuelos ${ }^{18, a}$, A.H. Ball ${ }^{17}$, E. Barberio ${ }^{8}$, R.J. Barlow ${ }^{16}$, R. Bartoldus ${ }^{3}$, J.R. Batley ${ }^{5}$, J. Bechtluft ${ }^{14}$, C. Beeston ${ }^{16}$, T. Behnke ${ }^{8}$, A.N. Bell ${ }^{1}$, K.W. Bell ${ }^{20}$, G. Bella ${ }^{23}$, S. Bentvelsen ${ }^{8}$, P. Berlich ${ }^{10}$, S. Bethke ${ }^{14}$, O. Biebel ${ }^{14}$, V. Blobel ${ }^{8}$, I.J. Bloodworth ${ }^{1}$, J.E. Bloomer ${ }^{1}$, M. Bobinski ${ }^{10}$, P. Bock ${ }^{11}$, H.M. Bosch ${ }^{11}$, M. Boutemeur ${ }^{34}$, B.T. Bouwens ${ }^{12}$,

S. Braibant ${ }^{12}$, R.M. Brown ${ }^{20}$, H.J. Burckhart ${ }^{8}$, C. Burgard ${ }^{8}$, R. Bürgin ${ }^{10}$, P. Capiluppi ${ }^{2}$, R.K. Carnegie ${ }^{6}$, A.A. Carter ${ }^{13}$, J.R. Carter ${ }^{5}$, C.Y. Chang ${ }^{17}$, C. Charlesworth ${ }^{6}$, D.G. Charlton ${ }^{1, b}$, D. Chrisman ${ }^{4}$, S.L. Chu ${ }^{4}$, P.E.L.Clarke ${ }^{15}$, I. Cohen ${ }^{23}$, J.E. Conboy ${ }^{15}$, O.C. Cooke ${ }^{16}$, M. Cuffiani ${ }^{2}$, S. Dado ${ }^{22}$, C. Dallapiccola ${ }^{17}$, G.M. Dallavalle ${ }^{2}$, S. De Jong ${ }^{12}$, L.A. del Pozo ${ }^{8}$, K. Desch ${ }^{3}$, M.S. Dixit ${ }^{7}$, E. do Couto e Silva ${ }^{12}$, M. Doucet ${ }^{18}$, E. Duchovni ${ }^{26}$, G. Duckeck ${ }^{34}$, I.P. Duerdoth ${ }^{16}$, J.E.G. Edwards ${ }^{16}$, P.G. Estabrooks $^{6}$, H.G. Evans ${ }^{9}$, M. Evans ${ }^{13}$, F. Fabbri ${ }^{2}$, P. Fath ${ }^{11}$, F. Fiedler ${ }^{12}$, M. Fierro ${ }^{2}$, H.M. Fischer ${ }^{3}$, R. Folman ${ }^{26}$, D.G. Fong ${ }^{17}$, M. Foucher ${ }^{17}$, A. Fürtjes $^{8}$, P. Gagnon ${ }^{7}$, A. Gaidot ${ }^{21}$, J.W. Gary ${ }^{4}$, J. Gascon ${ }^{18}$, S.M. Gascon-Shotkin ${ }^{17}$, N.I. Geddes ${ }^{20}$, C. Geich-Gimbel ${ }^{3}$, F.X. Gentit ${ }^{21}$, T. Geralis ${ }^{20}$, G. Giacomelli ${ }^{2}$, P. Giacomelli ${ }^{4}$, R. Giacomelli ${ }^{2}$, V. Gibson ${ }^{5}$, W.R. Gibson ${ }^{13}$, D.M. Gingrich ${ }^{30, a}$, D. Glenzinski ${ }^{9}$, J. Goldberg ${ }^{22}$, M.J. Goodrick ${ }^{5}$, W. Gorn ${ }^{4}$, C. Grandi ${ }^{2}$, E. Gross ${ }^{26}$, M. Gruwé ${ }^{8}$, C.Hajdu ${ }^{32}$, G.G. Hanson ${ }^{12}$, M.Hansroul ${ }^{8}$, M.Hapke ${ }^{13}$, C.K. Hargrove ${ }^{7}$, P.A.Hart ${ }^{9}$, C.Hartmann ${ }^{3}$, M. Hauschild ${ }^{8}$, C.M.Hawkes ${ }^{5}$, R.Hawkings ${ }^{8}$, R.J.Hemingway ${ }^{6}$, G. Herten ${ }^{10}$, R.D. Heuer ${ }^{8}$, M.D. Hildreth ${ }^{8}$, J.C. Hill ${ }^{5}$, S.J. Hillier ${ }^{1}$, T. Hilse ${ }^{10}$, P.R.Hobson ${ }^{25}$, R.J.Homer ${ }^{1}$, A.K.Honma ${ }^{28, a}$, D. Horváth ${ }^{32, c}$, R. Howard ${ }^{29}$, R.E.Hughes-Jones ${ }^{16}$, D.E. Hutchcroft ${ }^{5}$, P. Igo-Kemenes ${ }^{11}$, D.C. Imrie ${ }^{25}$, M.R. Ingram ${ }^{16}$, K. Ishii ${ }^{4}$, A. Jawahery ${ }^{17}$, P.W. Jeffreys ${ }^{20}$, H. Jeremie ${ }^{18}$, M. Jimack ${ }^{1}$, A. Joly ${ }^{18}$, C.R. Jones ${ }^{5}$, G. Jones ${ }^{16}$, M. Jones ${ }^{6}$, R.W.L. Jones ${ }^{8}$, U. Jost ${ }^{11}$, P. Jovanovic ${ }^{1}$, T.R. Junk ${ }^{8}$, D. Karlen ${ }^{6}$, K. Kawagoe ${ }^{24}$, T. Kawamoto ${ }^{24}$, R.K. Keeler ${ }^{28}$, R.G. Kellogg ${ }^{17}$, B.W. Kennedy ${ }^{20}$, B.J. King ${ }^{8}$, J. Kirk ${ }^{29}$, S. Kluth ${ }^{8}$, T. Kobayashi ${ }^{24}$, M. Kobel ${ }^{10}$, D.S. Koetke ${ }^{6}$, T.P. Kokott ${ }^{3}$, M. Kolrep ${ }^{10}$, S. Komamiya ${ }^{24}$, R. Kowalewski ${ }^{8}$, T. Kress ${ }^{11}$, P. Krieger ${ }^{6}$, J. von Krogh ${ }^{11}$, P. Kyberd ${ }^{13}$, G.D. Lafferty $^{16}$, H. Lafoux ${ }^{21}$, R. Lahmann ${ }^{17}$, W.P. Lai ${ }^{19}$, D. Lanske ${ }^{14}$, J. Lauber ${ }^{15}$, S.R. Lautenschlager ${ }^{31}$, J.G. Layter ${ }^{4}$, D. Lazic ${ }^{22}$, A.M. Lee ${ }^{31}$, E. Lefebvre ${ }^{18}$, D. Lellouch ${ }^{26}$, J. Letts $^{2}$, L. Levinson ${ }^{26}$, C. Lewis ${ }^{15}$, S.L. Lloyd ${ }^{13}$, F.K. Loebinger ${ }^{16}$, G.D. Long ${ }^{17}$, M.J. Losty ${ }^{7}$, J. Ludwig ${ }^{10}$, A. Malik ${ }^{21}$, M. Mannelli ${ }^{8}$, S. Marcellini ${ }^{2}$, C. Markus ${ }^{3}$, A.J. Martin ${ }^{13}$, J.P. Martin ${ }^{18}$, G. Martinez ${ }^{17}$, T. Mashimo ${ }^{24}$, W. Matthews ${ }^{25}$, P. Mättig ${ }^{3}$, W.J. McDonald ${ }^{30}$, J. McKenna ${ }^{29}$, E.A. Mckigney ${ }^{15}$, T.J. McMahon ${ }^{1}$, A.I. McNab ${ }^{13}$, R.A. McPherson ${ }^{8}$, F. Meijers ${ }^{8}$, S. Menke ${ }^{3}$, F.S. Merritt ${ }^{9}$, H. Mes ${ }^{7}$, J. Meyer ${ }^{27}$, A. Michelini ${ }^{2}$, G. Mikenberg ${ }^{26}$, D.J. Miller ${ }^{15}$, R. Mir ${ }^{26}$, W. Mohr ${ }^{10}$, A. Montanari ${ }^{2}$, T. Mori ${ }^{24}$, M. Morii ${ }^{24}$, U. Müller ${ }^{3}$, K. Nagai ${ }^{26}$, I. Nakamura ${ }^{24}$, H.A. Neal ${ }^{8}$, B. Nellen ${ }^{3}$, B. Nijjhar ${ }^{16}$, R. Nisius ${ }^{8}$, S.W. O’Neale ${ }^{1}$, F.G.Oakham ${ }^{7}$, F. Odorici ${ }^{2}$, H.O. Ogren ${ }^{12}$, T. Omori ${ }^{24}$, M.J. Oreglia ${ }^{9}$, S. Orito ${ }^{24}$, J. Pálinkás ${ }^{33, d}$, G. Pásztor ${ }^{32}$, J.R. Pater ${ }^{16}$, G.N.Patrick ${ }^{20}$, J. Patt ${ }^{10}$, M.J. Pearce ${ }^{1}$, S. Petzold ${ }^{27}$, P. Pfeifenschneider ${ }^{14}$, J.E. Pilcher ${ }^{9}$, J. Pinfold ${ }^{30}$, D.E. Plane ${ }^{8}$, P. Poffenberger ${ }^{28}$, B. Poli ${ }^{2}$, A.Posthaus ${ }^{3}$, H. Przysiezniak ${ }^{30}$, D.L. Rees ${ }^{1}$, D. Rigby ${ }^{1}$, S.A. Robins ${ }^{13}$, N. Rodning ${ }^{30}$, J.M. Roney ${ }^{28}$, A. Rooke ${ }^{15}$, E. Ros ${ }^{8}$,

A.M. Rossi ${ }^{2}$, M. Rosvick ${ }^{28}$, P. Routenburg ${ }^{30}$, Y. Rozen $^{22}$, K. Runge $^{10}$, O. Runolfsson ${ }^{8}$, U. Ruppel ${ }^{14}$, D.R. Rust ${ }^{12}$, R. Rylko ${ }^{25}$, K. Sachs ${ }^{10}$, E.K.G.Sarkisyan ${ }^{23}$, M.Sasaki ${ }^{24}$, C.Sbarra ${ }^{2}$,

A.D.Schaile ${ }^{34}$, O.Schaile ${ }^{34}$, F. Scharf ${ }^{3}$, P.Scharff-Hansen ${ }^{8}$, P.Schenk ${ }^{4}$, B. Schmitt ${ }^{8}$, S.Schmitt ${ }^{11}$, M.Schröder ${ }^{8}$, H.C.Schultz-Coulon ${ }^{10}$, M.Schulz ${ }^{8}$, M.Schumacher ${ }^{3}$, P.Schütz ${ }^{3}$, W.G.S $\operatorname{cott}^{20}$, T.G.Shears ${ }^{16}$, B.C.Shen ${ }^{4}$, C.H. Shepherd-Themistocleous ${ }^{27}$, P. Sherwood ${ }^{15}$, G.P.Siroli ${ }^{2}$, A.Sittler ${ }^{27}$, A.Skillman ${ }^{15}$, A.Skuja ${ }^{17}$, A.M.Smith ${ }^{8}$, T.J.Smith ${ }^{28}$, G.A.Snow ${ }^{17}$, 
R. Sobie ${ }^{28}$, S. Söldner-Rembold ${ }^{10}$, R.W.Springer ${ }^{30}$, M.Sproston ${ }^{20}$, A.Stahl ${ }^{3}$, M. Starks ${ }^{12}$, M. Steiert ${ }^{11}$, K. Stephens ${ }^{16}$, J. Steuerer ${ }^{27}$, B. Stockhausen ${ }^{3}$, D. Strom ${ }^{19}$, F. Strumia ${ }^{8}$, P. Szymanski ${ }^{20}$, R. Tafirout ${ }^{18}$, S.D. Talbot ${ }^{1}$, S. Tanaka ${ }^{24}$, P. Taras ${ }^{18}$, S. Tarem ${ }^{22}$, M. Tecchio ${ }^{8}$,

M. Thiergen ${ }^{10}$, M.A. Thomson ${ }^{8}$, E. von Törne ${ }^{3}$, S. Towers ${ }^{6}$, T. Tsukamoto ${ }^{24}$, E. Tsur ${ }^{23}$, A.S. Turcot ${ }^{9}$, M.F. Turner-Watson ${ }^{8}$, P. Utzat ${ }^{11}$, R. Van Kooten ${ }^{12}$, G. Vasseur ${ }^{21}$, M. Verzocchi ${ }^{10}$, P. Vikas ${ }^{18}$, M.Vincter ${ }^{28}$, E.H. Vokurka ${ }^{16}$, F.Wäckerle ${ }^{10}$, A. Wagner $^{27}$, C.P. Ward ${ }^{5}$, D.R. Ward ${ }^{5}$, J.J.Ward ${ }^{15}$, P.M. Watkins ${ }^{1}$, A.T. Watson ${ }^{1}$, N.K. Watson ${ }^{7}$, P. Weber ${ }^{6}$, P.S. Wells ${ }^{8}$, N. Wermes ${ }^{3}$, J.S. White ${ }^{28}$, B. Wilkens ${ }^{10}$, G.W.Wilson ${ }^{27}$, J.A.Wilson ${ }^{1}$, G. Wolf ${ }^{26}$, S. Wotton ${ }^{5}$, T.R. Wyatt ${ }^{16}$, S. Yamashita ${ }^{24}$, G. Yekutielii ${ }^{26}$, V. Zacek ${ }^{18}$,

${ }^{1}$ School of Physics and Space Research, University of Birmingham, Birmingham B15 2TT, UK ${ }^{2}$ Dipartimento di Fisica dell' Università di Bologna and INFN, I-40126 Bologna, Italy

${ }^{3}$ Physikalisches Institut, Universität Bonn, D-53115 Bonn, Germany

${ }^{4}$ Department of Physics, University of California, Riverside CA 92521, USA

${ }^{5}$ Cavendish Laboratory, Cambridge CB3 0HE, UK

${ }^{6}$ Ottawa-Carleton Institute for Physics, Department of Physics, Carleton University, Ottawa, Ontario K1S 5B6, Canada

${ }^{7}$ Centre for Research in Particle Physics, Carleton University, Ottawa, Ontario K1S 5B6, Canada

${ }^{8}$ CERN, European Organisation for Particle Physics, CH-1211 Geneva 23, Switzerland

${ }^{9}$ Enrico Fermi Institute and Department of Physics, University of Chicago, Chicago IL 60637, USA

${ }^{10}$ Fakultät für Physik, Albert Ludwigs Universität, D-79104 Freiburg, Germany

${ }^{11}$ Physikalisches Institut, Universität Heidelberg, D-69120 Heidelberg, Germany

${ }^{12}$ Indiana University, Department of Physics, Swain Hall West 117, Bloomington IN 47405, USA

${ }^{13}$ Queen Mary and Westfield College, University of London, London E1 4NS, UK

${ }^{14}$ Technische Hochschule Aachen, III Physikalisches Institut, Sommerfeldstrasse 26-28, D-52056 Aachen, Germany

${ }^{15}$ University College London, London WC1E 6BT, UK

${ }^{16}$ Department of Physics, Schuster Laboratory, The University, Manchester M13 9PL, UK

${ }^{17}$ Department of Physics, University of Maryland, College Park, MD 20742, USA

${ }^{18}$ Laboratoire de Physique Nucléaire, Université de Montréal, Montréal, Quebec H3C 3J7, Canada

${ }^{19}$ University of Oregon, Department of Physics, Eugene OR 97403, USA

${ }^{20}$ Rutherford Appleton Laboratory, Chilton, Didcot, Oxfordshire OX11 0QX, UK

${ }^{21}$ CEA, DAPNIA/SPP, CE-Saclay, F-91191 Gif-sur-Yvette, France

${ }^{22}$ Department of Physics, Technion-Israel Institute of Technology, Haifa 32000, Israel

${ }^{23}$ Department of Physics and Astronomy, Tel Aviv University, Tel Aviv 69978, Israel

${ }^{24}$ International Centre for Elementary Particle Physics and Department of Physics, University of Tokyo, Tokyo 113, and Kobe University, Kobe 657, Japan

${ }^{25}$ Brunel University, Uxbridge, Middlesex UB8 3PH, UK

${ }^{26}$ Particle Physics Department, Weizmann Institute of Science, Rehovot 76100, Israel

${ }^{27}$ Universität Hamburg/DESY, II Institut für Experimental Physik, Notkestrasse 85, D-22607

Hamburg, Germany

${ }^{28}$ University of Victoria, Department of Physics, P O Box 3055, Victoria BC V8W 3P6, Canada 
${ }^{29}$ University of British Columbia, Department of Physics, Vancouver BC V6T 1Z1, Canada ${ }^{30}$ University of Alberta, Department of Physics, Edmonton AB T6G 2J1, Canada

${ }^{31}$ Duke University, Dept of Physics, Durham, NC 27708-0305, USA

${ }^{32}$ Research Institute for Particle and Nuclear Physics, H-1525 Budapest, P O Box 49, Hungary ${ }^{33}$ Institute of Nuclear Research, H-4001 Debrecen, P O Box 51, Hungary

${ }^{34}$ Ludwigs-Maximilians-Universität München, Sektion Physik, Am Coulombwall 1, D-85748 Garching, Germany

${ }^{a}$ and at TRIUMF, Vancouver, Canada V6T $2 \mathrm{~A} 3$

${ }^{b}$ and Royal Society University Research Fellow

${ }^{c}$ and Institute of Nuclear Research, Debrecen, Hungary

${ }^{d}$ and Department of Experimental Physics, Lajos Kossuth University, Debrecen, Hungary 


\section{Introduction}

Electroweak measurements involving heavy quarks have become a subject of extensive study at LEP in recent years [1]. An important goal of these measurements is the precise determination of the coupling constants of the weak currents and their comparison with the predictions of the standard model. The angular distributions of the final state fermions provide a probe of these coupling constants. In $\mathrm{e}^{+} \mathrm{e}^{-}$collisions at LEP the different strengths of the left-handed and the right-handed components of the neutral weak current result in an asymmetry of the differential production cross-section relative to the direction of the initial state electron. In addition, the electromagnetic and the neutral weak currents interfere. On the peak of the $Z^{0}$ resonance this latter interference vanishes to first order, but it becomes more and more important at energies away from the $\mathrm{Z}^{0}$ pole. In the standard model, to lowest order, the differential cross-section in $\cos \theta^{1}$ for the process $\mathrm{e}^{+} \mathrm{e}^{-} \rightarrow \mathrm{f} \overline{\mathrm{f}}$, where $\mathrm{f}$ denotes any fermion, can be written as:

$$
\frac{\mathrm{d} \sigma}{\mathrm{d} \cos \theta} \propto 1+\cos ^{2} \theta+\frac{8}{3} A_{\mathrm{FB}}^{\mathrm{f}} \cos \theta
$$

On the pole of the $\mathrm{Z}^{0}$ resonance, for quark flavour $\mathrm{Q}$, the forward-backward asymmetry, $A_{\mathrm{FB}}^{\mathrm{Q}, 0}$, can be expressed as

$$
A_{\mathrm{FB}}^{\mathrm{Q}, 0}=\frac{3}{4} \mathcal{A}_{\mathrm{e}} \mathcal{A}_{\mathrm{Q}}
$$

where $\mathcal{A}_{\mathrm{e}}$ and $\mathcal{A}_{\mathrm{Q}}$ are functions of the electroweak vector- and axial-vector coupling constants $g_{\mathrm{V}}^{\mathrm{f}}$ and $g_{\mathrm{A}}^{\mathrm{f}}$ :

$$
\mathcal{A}_{\mathrm{f}}=\frac{2 g_{\mathrm{V}}^{\mathrm{f}} g_{\mathrm{A}}^{\mathrm{f}}}{\left(g_{\mathrm{V}}^{\mathrm{f}}\right)^{2}+\left(g_{\mathrm{A}}^{\mathrm{f}}\right)^{2}} .
$$

Often the standard model is expressed as a function of the weak mixing angle $\sin ^{2} \theta_{\mathrm{W}}$, which is related to the axial and vector coupling constants through the relation

$$
\sin ^{2} \theta_{\mathrm{W}}^{\mathrm{eff}, \mathrm{f}} \equiv \frac{1}{4\left|q_{\mathrm{f}}\right|}\left(1-\frac{g_{\mathrm{V}}^{\mathrm{f}}}{g_{A}^{\mathrm{f}}}\right) .
$$

Here $q_{\mathrm{f}}$ denotes the charge of the fermion f. Measuring the forward-backward asymmetries therefore allows a determination of the weak mixing angle.

In practice a number of experimental difficulties exist, which complicate the transition from the measured asymmetries in eq. 1 to the pole asymmetries defined in eq. 2. Since the direction of the initial quark is not directly measurable it is usually approximated by the thrust direction in the event. This makes the measurement sensitive to initial and final state photon radiation, and to QCD effects. These are corrected using theoretical predictions, which will be briefly discussed at the end of the paper.

In this paper measurements of the forward-backward asymmetries in the processes $\mathrm{e}^{+} \mathrm{e}^{-} \rightarrow$ $c \bar{c}$ and $\mathrm{e}^{+} \mathrm{e}^{-} \rightarrow \mathrm{b} \overline{\mathrm{b}}$ are presented. Decays of the $\mathrm{Z}^{0}$ into $c \overline{\mathrm{c}}$ and $\mathrm{b} \overline{\mathrm{b}}$ quark pairs are tagged using $\mathrm{D}^{* \pm}, \mathrm{D}^{0}$ and $\mathrm{D}^{ \pm}$mesons in a number of different decay modes. The forward-backward asymmetries of charm and bottom quarks are extracted via an unbinned maximum likelihood fit. The charm and bottom contributions to the signal are separated by calculating probabilities individually for each event from lifetime and jet shape information. The fit is performed for

\footnotetext{
${ }^{1}$ Here $\theta$ denotes the angle between the incoming electron and the outgoing fermion, $f$, in the process $\mathrm{e}^{+} \mathrm{e}^{-} \rightarrow \mathrm{f} \overline{\mathrm{f}}$.
} 
three energy ranges spanning approximately $M_{\mathrm{Z}^{0}}-3 \mathrm{GeV}$ to $M_{\mathrm{Z}^{0}}+3 \mathrm{GeV}$, where $M_{\mathrm{Z}^{0}}$ is the mass of the $\mathrm{Z}^{0}$ boson.

The paper is structured as follows. After a presentation of the techniques used to reconstruct the different D states, a method is described to separate the flavour composition of the D meson sample, based mainly on lifetime information. Subsequently the technique for the determination of the asymmetries is discussed.

\section{OPAL Detector}

A complete description of the OPAL detector can be found in references [2]. Only the components relevant to the analysis presented here are summarised. Tracking of charged particles is performed in a central detector, consisting, from the beampipe outwards, of two layers of silicon microvertex detectors, a high precision vertex drift chamber, a large volume jet chamber, and a set of drift chambers which measure the coordinates of tracks along the direction of the beam line ( $z$-chambers). The central detector is contained inside a solenoidal magnet producing a homogeneous field of $0.435 \mathrm{~T}$ throughout the tracking volume. Tracking using the combined detectors is possible over nearly the full solid angle, $|\cos \theta|<0.98$. High precision reconstruction of secondary vertices is possible using the silicon microvertex detector, which covers polar angles $|\cos \theta| \leq 0.85$ with at least one layer of silicon detectors, and $|\cos \theta|<0.77$ with two layers.

The magnet coil is surrounded by a time-of-flight scintillation counter array and a lead glass electromagnetic calorimeter. A presampler is installed between these components which allows an improvement of the measurement of the longitudinal shower development and spatial resolution for showers which started in the magnet coil of the detector, and provides an additional space point on tracks leaving the central tracking system. The magnet return yoke is instrumented with nine layers of limited streamer tubes and serves as a hadronic calorimeter. Outside the hadron calorimeter, $93 \%$ of the solid angle is covered by at least 2, usually 4 , layers of muon chambers.

Particle identification for charged particles is performed using the specific energy loss, $\mathrm{d} E / \mathrm{d} x$, in the jet chamber. Electrons and muons are identified with the help of information from the calorimeters and muon chambers.

Charged tracks and electromagnetic energy clusters unassociated with tracks are grouped into jets using the JADE jet finder and the E0 recombination scheme [3] with a cutoff value of $x_{\min }=49 \mathrm{GeV}^{2}$. The primary vertex in a collision is reconstructed from the charged tracks in the event and constrained in addition by the known average beam position and size of the $\mathrm{e}^{+} \mathrm{e}^{-}$interaction point.

Secondary vertices are reconstructed in the event by an iterative procedure [4]. All tracks in a jet which pass loose track quality criteria are used to form a common vertex. Tracks are removed from the vertex fit if they contribute more than 4 to the $\chi^{2}$ of the fit. This is continued until either no track contributes more than 4 to the $\chi^{2}$, or until only one track remains, in which case the vertex finder fails for this jet. A decay length is calculated between this vertex and the primary vertex in the event, constrained by the direction of the jet in which the vertex was found, in the plane perpendicular to the beam direction. Finally, the decay length significance $\delta$ for this vertex (i.e. the decay length divided by its error) is calculated. 


\section{Hadronic Event Selection and Simulation}

Hadronic $\mathrm{Z}^{0}$ decays are selected by placing requirements on the number of reconstructed charged tracks and the energy deposited in the calorimeter. A detailed description of the criteria is given in [5]. The analysis employs about 4 million hadronic decays of the $\mathrm{Z}^{0}$ collected with the OPAL detector in the vicinity of the $\mathrm{Z}^{0}$ resonance between 1990 and 1995. Of these data roughly $13.4 \%$ have been collected either below or above the maximum of the resonance.

To check procedures and investigate possible biases, 4 million hadronic decays of the $\mathrm{Z}^{0}$ have been simulated using the JETSET Monte Carlo model [6] with parameters tuned to represent LEP data well [7]. Several special samples have been used to study specific decays, which correspond to an additional 5 million hadronic $\mathrm{Z}^{0}$ decays. In all samples heavy quark fragmentation has been implemented using the model of Peterson et al. [8]. All samples have been passed through a detailed simulation of the OPAL detector [9] before being analysed using the same programs as for data.

\section{Selection of D Candidates}

Three different $\mathrm{D}$ meson states are used in this paper as tags for charm and bottom: the ground state pseudoscalar $\mathrm{D}$ mesons ${ }^{2} \mathrm{D}^{0}$ and $\mathrm{D}^{+}$, and the vector meson $\mathrm{D}^{*+}$. After describing the requirements placed on tracks to be included in the analysis, the reconstruction and the procedures used in the identification of charged $\mathrm{D}^{*}$ mesons are discussed, followed by the analogous discussion for $\mathrm{D}^{0}$ and $\mathrm{D}^{+}$mesons. Additional details on $\mathrm{D}$ reconstruction with the OPAL detector can be found in $[10-12]$.

Tracks are accepted for the reconstruction if they satisfy loose track quality requirements $\left(\left|d_{0}\right|<5 \mathrm{~mm} ;\left|z_{0}\right|<20 \mathrm{~cm} ; p_{\perp}>250 \mathrm{MeV} ; n_{\mathrm{CJ}}>40\right)$. Here $d_{0}$ is the distance of closest approach in the plane perpendicular to the beam axis, between the primary vertex and the track, $z_{0}$ is the distance along the beam at this point, $p_{\perp}$ is the momentum component perpendicular to the beam, and $n_{\mathrm{CJ}}$ is the number of hits on the track recorded in the jet chamber. Tracks are also required to be well reconstructed, by requiring that their polar angle $\theta$ be measured in at least two components of the tracking system, one of which must be the jet chamber.

\section{1 $\quad \mathrm{D}^{*+}$ Reconstruction}

For this analysis $\mathrm{D}^{*+}$ mesons are reconstructed in five different decay channels:

$$
\begin{aligned}
& \mathrm{D}^{*+} \rightarrow \mathrm{D}^{0} \pi^{+} \\
& \longrightarrow \mathrm{K}^{-} \pi^{+} \quad \text { "3 prong", } \\
& \hookrightarrow \mathrm{K}^{-} \pi^{+} \pi^{0} \quad \text { "satellite", } \\
& \longrightarrow \mathrm{K}^{-} \pi^{+} \pi^{-} \pi^{+} \quad \text { "5 prong" , } \\
& \longrightarrow \mathrm{K}^{-} \mathrm{e}^{+} \nu_{\mathrm{e}} \quad \text { "electron", } \\
& \rightarrow \mathrm{K}^{-} \mu^{+} \nu_{\mu} \quad \text { "muon". }
\end{aligned}
$$

\footnotetext{
${ }^{2}$ Throughout this paper charge conjugate modes are always implicitly included.
} 
No attempt is made to reconstruct the $\pi^{0}$ in the satellite channel, nor the neutrino direction or energy in the electron and muon channels. The last two channels are referred to as "semileptonic" channels in the following text. Electrons are identified based on the energy loss in the central drift chamber and the energy deposition in the electromagnetic calorimeter. An artificial neural network trained on simulated events is used to perform the selection [13]. Electrons from photon conversions are rejected as in [14]. Muon candidates are identified by associating tracks found in the central tracking system with tracks in the muon chambers [14]. No momentum cut is applied since knowledge of the lepton purity is not required in the subsequent analysis.

A number of tracks appropriate for the selected channel are combined to form a $\mathrm{D}^{0}$ candidate and their invariant mass is calculated. These cuts are given in table 1 and explained below. Only candidates with the correct charge combinations are retained. Candidates are selected if the reconstructed mass lies within the expected range for that channel. After adding a further track as a possible pion from the $\mathrm{D}^{*+}$ decay, the combined mass is calculated and the candidate is selected if the mass difference $\Delta M=M_{\mathrm{D}^{*+}}-M_{\mathrm{D}^{0}}$ is within certain limits. Note that $\Delta M$ is always to be understood as the effective mass difference, calculated only from the visible tracks of the candidate. This latter requirement is particularly efficient in suppressing background, because the small mass difference between the $\mathrm{D}^{*+}$ and the $\mathrm{D}^{0}$ places true $\mathrm{D}^{*+}$ decays very close to the kinematic threshold in $\Delta M$ at $0.139 \mathrm{GeV}$. Very little phase space remains for background events, resulting in a good signal to background ratio.

At low values of the $\mathrm{D}^{*+}$ scaled energy ${ }^{3}, x_{\mathrm{D}^{*+}}=E_{\mathrm{D}^{*+}}^{\text {calc }} / E_{\text {beam }}$, background, especially from pions produced in the fragmentation, is very high. Many of the background kaon candidates are such fragmentation pions. Their contribution is reduced by using the particle identification power of the OPAL detector to enrich the sample in true kaons. A probability, $W_{\mathrm{d} E / \mathrm{d} x}^{\mathrm{KK}}$, is calculated for a kaon candidate, that the measured specific energy loss, $\mathrm{d} E / \mathrm{d} x$, for a track is compatible with having been produced by a kaon. The probability $W_{\mathrm{d} E / \mathrm{d} x}^{\mathrm{KK}}$ is signed, i.e. positive and negative probabilities for values above and below the expected value, respectively.

Background to the sample is further reduced by cutting on the helicity angle $\theta^{*}$, measured between the direction of the $\mathrm{D}^{0}$ candidate in the laboratory frame and the direction of the kaon in the rest frame of the $D^{0}$ candidate. True $D^{0}$ decays are expected to be uniformly distributed in $\cos \theta^{*}$, while background displays pronounced peaks at $\cos \theta^{*}=-1$ and, particularly at low $x_{\mathrm{D}^{*+}}, \cos \theta^{*}=+1$.

The invariant mass spectra for the five reconstructed $\mathrm{D}^{*+}$ channels are presented in figure 1 . Shown is the mass difference $\Delta M$ between the $\mathrm{D}^{*+}$ and the $\mathrm{D}^{0}$ candidate. In all channels clear signals are visible.

\section{$4.2 \quad \mathrm{D}^{0}$ and $\mathrm{D}^{+}$Reconstruction}

The $\mathrm{D}^{0}$ and $\mathrm{D}^{+}$mesons are identified in the following decay modes:

$$
\begin{aligned}
& \mathrm{D}^{0} \rightarrow \mathrm{K}^{-} \pi^{+} \\
& \mathrm{D}^{+} \rightarrow \mathrm{K}^{-} \pi^{+} \pi^{+}
\end{aligned}
$$

\footnotetext{
${ }^{3}$ In this paper any reference to the scaled energy $x$ of a $\mathrm{D}$ candidate is to be understood as being to the calculated energy of the D, $E_{\mathrm{D}}$, obtained from the reconstructed tracks, without correcting for missing or wrongly associated tracks, divided by the beam energy $E_{\text {beam }}$.
} 


\begin{tabular}{|c|c|c|c|c|c|}
\hline \hline cut & $x$ range & 3 prong & semileptonic & satellite & 5 prong \\
\hline \hline$x_{\mathrm{D}^{*+}}$ & & $0.2-1.0$ & $0.2-1.0$ & $0.2-1.0$ & $0.5-1.0$ \\
\hline$M_{\mathrm{D}^{0}}[\mathrm{GeV}]$ & full & $1.79-1.94$ & $1.20-1.80$ & $1.41-1.77$ & $1.79-1.94$ \\
\hline$\Delta M[\mathrm{GeV}]$ & full & $0.142-0.149$ & $0.140-0.162$ & $0.141-0.151$ & $0.142-0.149$ \\
\hline $\cos \theta^{*}$ & $<0.5$ & \multicolumn{3}{|c}{} & - \\
& $>0.5$ & & $-0.8-0.8$ & $-0.9-1.0$ \\
\hline$\left|W_{\mathrm{d} E / \mathrm{d} x}^{\mathrm{KK}}\right|$ & $<0.5$ & \multicolumn{5}{|c}{$>-1.0$} & - \\
\hline \hline
\end{tabular}

Table 1: List of cuts used in the $\mathrm{D}^{*+}$ reconstruction. Note that both the scaled energy $x_{\mathrm{D}}$ and the mass difference $\Delta M$ are effective quantities, calculated from the reconstructed tracks only. The exact meaning of the different quantities is explained in the text.

The reconstruction of these $\mathrm{D}$ meson states is similar to that for the $\mathrm{D}^{*+}$ mesons. It follows very closely the method described in [12]. The invariant mass is calculated from the appropriate number of tracks which pass the quality requirements. $\mathrm{A}^{+}$candidate is rejected if the mass difference $\Delta M$, calculated under the assumption that the three tracks form a $\mathrm{D}^{*+}$ candidate, is below $0.16 \mathrm{GeV}$. To suppress combinatorial background, and to allow a good measurement of the specific energy loss $\mathrm{d} E / \mathrm{d} x$, candidates are required to have at least $30 \%$ of the beam energy, and the individual track momenta are required to be above $1.5 \mathrm{GeV}$ for a kaon, and $0.5 \mathrm{GeV}$ for a pion. The long lifetime of the weakly decaying $\mathrm{D}$ mesons is used to enrich the signal purity. A vertex is calculated in the $r$ - $\phi$ plane from the tracks forming the $\mathrm{D}$ candidate. The distance $d$ between this vertex and the primary event vertex is calculated. A candidate is accepted if this distance exceeds $500 \mu \mathrm{m}$ for a $\mathrm{D}^{0}$ candidate, or $800 \mu \mathrm{m}$ for a $\mathrm{D}^{+}$candidate. Good vertex reconstruction quality is ensured by requiring that at least one (for the $\mathrm{D}^{0}$ ) or two (for the $\mathrm{D}^{+}$) tracks have at least one hit in the silicon microvertex detector. To stay within the acceptance of this detector, all tracks have to be within $|\cos \theta|<0.85$. For the $\mathrm{D}^{+}$, where the three tracks in the decay allow a meaningful calculation of a vertex $\chi^{2}$, events with badly reconstructed vertices are rejected by a loose cut on this quantity.

The number of multiple candidates and wrong particle type associations is reduced by placing requirements on the $\mathrm{d} E / \mathrm{d} x$ measurement. Kaon candidate tracks are required to be consistent with being a kaon with a probability $W_{\mathrm{d} E / \mathrm{d} x}^{\mathrm{KK}}$ exceeding $3 \%$ if their measured $\mathrm{d} E / \mathrm{d} x$ is above the value expected for a kaon, or exceeding $1 \%$ if the measured $\mathrm{d} E / \mathrm{d} x$ is below the expected value. Pion background in the kaon candidate sample is rejected by accepting only tracks whose probability $W_{\mathrm{d} E / \mathrm{d} x}^{\mathrm{K} \pi}$ that the kaon candidate track is consistent with a pion is less than $10 \%$. The purity of the pion candidates is increased by requiring that these tracks are consistent with a pion with a probability exceeding $1 \%$. The details of the cuts are listed in table 2. In figure 2 the invariant mass spectra for the $\mathrm{D}^{0}$ and $\mathrm{D}^{+}$channel are plotted.

\subsection{Multiple Candidate Rejection}

Since the goal of the analysis is the determination of the angular distribution of the primary quark, not that of reconstructed mesons, only one measurement of this quantity per event is needed. If more than one candidate meson is found per event, a hierarchy is used to select the best one: low background is preferred and low multiplicity final states are favoured over higher 


\begin{tabular}{|c|c|c|}
\hline \hline cut & $\mathrm{D}^{0}$ & $\mathrm{D}^{+}$ \\
\hline \hline$x_{\mathrm{D}}$ & $0.3-1.0$ & $0.3-1.0$ \\
\hline$M_{\mathrm{D}^{0}, \mathrm{D}^{+}}[\mathrm{GeV}]$ & $1.81-1.93$ & $1.81-1.93$ \\
\hline$\Delta M[\mathrm{GeV}]$ & - & $>\mathbf{0 . 1 6}$ \\
\hline$p_{\mathrm{K}}[\mathrm{GeV}]$ & $>1.5$ & $>1.5$ \\
$p_{\pi}[\mathrm{GeV}]$ & $>0.5$ & $>\mathbf{0 . 5}$ \\
\hline$\left|\cos \theta_{\text {cand }}\right|$ & $<\mathbf{0 . 8 5}$ & $<\mathbf{0 . 8 5}$ \\
\hline$d[\mu \mathrm{m}]$ & $>500$ & $>800$ \\
\hline$W_{\mathrm{d} E / \mathrm{d} x}^{\mathrm{KK}}$ & $>\mathbf{0 . 0 3}$ & $>\mathbf{0 . 0 3}$ \\
& $<-0.01$ & $<-0.01$ \\
\hline$\left|W_{\mathrm{d} E / \mathrm{d} x}^{\pi \pi}\right|$ & $>\mathbf{0 . 0 1}$ & $>\mathbf{0 . 0 1}$ \\
\hline$\left|W_{\mathrm{d} E / \mathrm{d} x}^{\mathrm{K}}\right|$ & $<0.1$ & $<\mathbf{0 . 1}$ \\
\hline \hline
\end{tabular}

Table 2: List of cuts used in the $\mathrm{D}^{0}$ and $\mathrm{D}^{+}$reconstruction. The exact meaning of the different quantities is explained in the text.

multiplicity ones. The hierarchy used, from best to worst, is: 3 prong, semileptonic, satellite, 5 prong, $\mathrm{D}^{0}, \mathrm{D}^{+}$. If more than one candidate is found within one $\mathrm{D}^{*+}$ channel, the one with the $\mathrm{D}^{0}$ mass closest to the nominal mass is taken (the mass used is $1.865 \mathrm{GeV}$ for the 3 prong, semileptonic and 5-prong decay, and $1.60 \mathrm{GeV}$ for the satellite [15]). In the $\mathrm{D}^{0}$ and $\mathrm{D}^{+}$decays, where a selection on the mass would result in a distortion of the invariant mass spectrum, the candidate with the highest $x_{\mathrm{D}}$ is selected. Overall the number of candidates is reduced by $34.6 \%$ by the multiple candidate rejection. After all cuts a total of 73870 candidates is found.

\subsection{Determination of the Signal Fraction}

For the purpose of the asymmetry analysis, a signal event is defined as any event that contains enough information to reconstruct correctly the charge and flavour of the primary quark. For the $\mathrm{D}^{*+}$ decays two classes of events contribute to the signal sample: events where a $\mathrm{D}$ meson has been completely reconstructed in one of the channels described, and events where a $\mathrm{D}^{*+}$ decay has only partially been found, but where the slow pion is correctly tagged. The latter have broader distributions in $\Delta M$, as is shown in figure 1 , especially in the 5 prong channel. In either case, because of the very distinct kinematics of the $D^{*+}$ decay, the events exhibit an enhancement in the $\Delta M$ spectra around $145 \mathrm{MeV}$. The fraction of events which is considered as signal for this analysis is determined with a background estimator, which uses a hemisphere mixing technique first described in [10]. In this estimator, the candidate for the slow pion is taken from the opposite hemisphere to the rest of the candidate tracks, and reflected through the origin before being used in the calculation of the invariant mass of the candidate. This method ensures that no correctly identified slow pions enter the background sample, and that the background shape does not exhibit any peaks in the interesting $\Delta M$ region. No requirements are put on the charge of the $\mathrm{D}^{0}$ candidate tracks, except that the sum of the charges of all tracks including the slow pion candidate should be \pm 1 . Monte Carlo studies have shown that this background estimator reliably models the shape of the background in all five channels. 
The background is determined by normalising the estimator distribution for $0.18 \mathrm{GeV}<$ $\Delta \mathrm{M}<0.20 \mathrm{GeV}(0.19 \mathrm{GeV}<\Delta \mathrm{M}<0.22 \mathrm{GeV}$ for the semileptonic channels $)$ to the sideband of the signal distribution and subtracting the normalised background from the signal. Candidates are counted within the $\Delta M$ ranges given in table 1 . Monte Carlo studies have shown that this method leads to an unbiased determination of the number of $\mathrm{D}^{*+}$ candidates in all channels.

The level of background in the $\mathrm{D}^{0}$ and $\mathrm{D}^{+}$channels is determined from a fit to the observed mass spectra with an empirically determined functional form for the background. The background to the $\mathrm{D}^{0}$ is described by a third degree polynomial, while for the $\mathrm{D}^{+}$an exponential convoluted with a Gaussian function is used. Contributions from satellite decays visible in the $\mathrm{D}^{0}$ mass spectrum at masses around $1.6 \mathrm{GeV}$ are accounted for by an additional Gaussian function. A slight asymmetry in the signal is allowed for by fitting different widths below and above the mean value. The normalisation, mean and both widths are determined in the fit. The total background is determined by integrating the fit function over the mass windows listed in table 2 .

There is background in both channels which distorts the expected signal shape. In the $\mathrm{D}^{0}$ channel the decays $\mathrm{D}^{0} \rightarrow \mathrm{K}^{+} \mathrm{K}^{-}$and $\mathrm{D}^{0} \rightarrow \pi^{+} \pi^{-}$contribute to the signal, if one of the final state particles is misidentified as either a pion or a kaon, thus faking a $K \pi$ final state. Since the charge correlation between the primary quark and the reconstructed charge is broken, their estimated contribution is subtracted from the sample. Simulated events are used to estimate the contamination from these two sources. They are found to contribute less than $1 \%$ to the final sample.

Backgrounds in the $\mathrm{D}^{+}$reconstruction are the decays of the $\mathrm{D}_{\mathrm{s}}$ meson into $\phi \pi^{+}$and $\mathrm{K}^{* 0} \mathrm{~K}^{+}$, both decaying into a final state $\mathrm{K}^{+} \mathrm{K}^{-} \pi^{+}$. Misidentifying a kaon as a pion will shift the mass peak to around the mass of the $\mathrm{D}^{+}$meson. Monte Carlo studies have shown that this background contributes approximately $10 \%$ to the $\mathrm{D}^{+}$signal.

The final number of $\mathrm{D}^{*+}, \mathrm{D}^{0}$ or $\mathrm{D}^{+}$events is determined by correcting the number of background subtracted events calculated above for these additional background contributions. This method has been tested using simulated events and has been found to reproduce the true number of $D$ mesons within the statistical precision of the test.

A potentially serious problem in all channels is the presence of background where the correlation between the primary quark charge and the charge $q$ tagged by the $\mathrm{D}$ candidate is preserved, but no true slow pion is present. These events will not be considered as signal by the above algorithm, but they will be asymmetric in $q \cos \theta$, thereby introducing an asymmetry into the background. Such correlations are expected since the sample of candidate tracks is enriched in true kaons using the $\mathrm{d} E / \mathrm{d} x$ cuts described. Many kaons are originating from the primary quark in the event, thereby preserving the charge correlation. Similar effects are expected for leptons. These events are present at levels of a few percent in all modes considered.

In table 3 the number of $\mathrm{D}^{*+}, \mathrm{D}^{0}$ and $\mathrm{D}^{+}$meson candidates, and the background, is shown for each channel, with statistical errors. Altogether $24195 \pm 150 \mathrm{D}^{*+}$ mesons and $8439 \pm 132$ $\mathrm{D}^{0}$ and $\mathrm{D}^{+}$mesons have been reconstructed after background subtraction, over backgrounds of $24664 \pm 150$ events and $16558 \pm 132$ events, respectively. 


\begin{tabular}{|c|c|c|c|c|c|c|c|}
\hline & \multicolumn{2}{|c|}{ 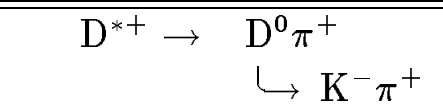 } & \multicolumn{2}{|c|}{ 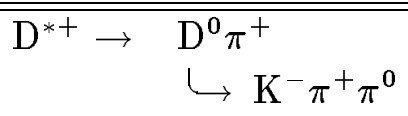 } & \multicolumn{3}{|c|}{$\begin{array}{ll}\mathrm{D}^{*+} \rightarrow & \mathrm{D}^{0} \boldsymbol{\pi}^{+} \\
& \stackrel{\longrightarrow}{\rightarrow} \mathrm{K}^{-} \boldsymbol{\pi}^{+} \boldsymbol{\pi}^{-} \boldsymbol{\pi}^{+}\end{array}$} \\
\hline$x_{\mathrm{D}}$ & $N_{\text {cand }}^{\text {obs }}$ & $N_{\text {bgd }}^{\text {est }}$ & $N_{\text {cand }}^{\text {obs }}$ & $N_{\text {bgd }}^{\text {est }}$ & $N_{\text {cand }}^{\text {obs }}$ & \multicolumn{2}{|c|}{$N_{\text {bgd }}^{\text {est }}$} \\
\hline $0.2-0.3$ & 3036 & $1716 \pm 39$ & 10372 & $7547 \pm 87$ & & & \\
\hline $0.3-0.4$ & 1825 & $704 \pm 25$ & 5131 & $2999 \pm \quad 54$ & & & \\
\hline $0.4-0.5$ & 1251 & $264 \pm 15$ & 3016 & $1273 \pm \quad 36$ & & & \\
\hline $0.5-0.6$ & 1556 & $411 \pm 18$ & 3636 & $1753 \pm \quad 40$ & 5417 & $3312 \pm$ & 52 \\
\hline $0.6-0.7$ & 927 & $169 \pm 11$ & 1687 & $725 \pm 25$ & 2156 & $1002 \pm$ & 28 \\
\hline $0.7-0.8$ & 520 & $76 \pm 7$ & 670 & $263 \pm 14$ & 908 & $298 \pm$ & 14 \\
\hline \multirow[t]{2}{*}{$0.8-1.0$} & 277 & $34 \pm 4$ & 208 & $97 \pm$ & 357 & $78 \pm$ & 6 \\
\hline & \multicolumn{2}{|c|}{$\begin{array}{ll}\mathrm{D}^{*+} \rightarrow & \mathrm{D}^{0} \pi^{+} \\
& \iota \mathrm{K}^{-} \ell^{+} \nu\end{array}$} & \multicolumn{2}{|c|}{$\overline{\mathrm{D}^{0} \rightarrow \quad \mathrm{K}^{-} \pi^{+}}$} & \multicolumn{3}{|c|}{$\mathrm{D}^{+} \rightarrow \mathrm{K}^{-} \pi^{+} \pi^{+}$} \\
\hline$x_{\mathrm{D}}$ & $N_{\text {cand }}^{\text {obs }}$ & $N_{\text {bgd }}^{\text {est }}$ & $N_{\text {cand }}^{\text {obs }}$ & $N_{\text {bgd }}^{\text {est }}$ & $N_{\text {cand }}^{\text {obs }}$ & \multicolumn{2}{|c|}{$N_{\text {bgd }}^{\text {est }}$} \\
\hline $0.2-0.3$ & 2217 & $1058 \pm 33$ & & & & & \\
\hline $0.3-0.4$ & 1405 & $475 \pm 22$ & 4102 & $2655 \pm \quad 52$ & 6314 & $5300 \pm$ & 72 \\
\hline $0.4-0.5$ & 861 & $212 \pm 15$ & 2513 & $1636 \pm \quad 51$ & 4089 & $2982 \pm$ & 55 \\
\hline $0.5-0.6$ & 829 & $99 \pm 14$ & 1711 & $770 \pm 28$ & 2318 & $1465 \pm$ & 38 \\
\hline $0.6-0.7$ & & & 985 & $455 \pm 21$ & 1289 & $669 \pm$ & 26 \\
\hline $0.7-0.8$ & 597 & $99 \pm 9$ & 514 & $224 \pm 15$ & 672 & $242 \pm$ & 16 \\
\hline $0.8-1.0$ & & & 224 & $92 \pm 10$ & 266 & $68 \pm$ & 8 \\
\hline
\end{tabular}

Table 3: Number of $\mathrm{D}$ mesons reconstructed in the different decay modes, in bins of the effective scaled energy of the candidates calculated from the reconstructed track momenta. Given are the number of D meson candidates, $N_{\text {cand }}^{\text {obs }}$, before background subtraction and the number of background events, $N_{\mathrm{bgd}}^{\text {est }}$. The error quoted on the estimated background is the statistical error only.

\section{Charmed Mesons in $c \bar{c}$ and $b \bar{b}$ Events}

In this analysis the $\mathrm{D}^{*+}, \mathrm{D}^{0}$ and $\mathrm{D}^{+}$mesons are used as tags for primary charm and bottom decays of the $\mathrm{Z}^{0}$. Both processes are expected to contribute roughly equally to the tagged sample, in addition to sizeable contributions from combinatorial background. Lifetime information and jet shape variables are used to separate the different sources of D mesons. First the level of background in the sample is determined, based on the $\mathrm{D}$ meson signal, as described in section 4.4, and subtracted. The remaining signal is composed essentially only of $c \bar{c}$ and $\mathrm{b} \overline{\mathrm{b}}$ events. Since $\mathrm{b} \overline{\mathrm{b}}$ events are significantly different in lifetime and jet shape properties from other events, the fraction of $b \bar{b}$ events in the sample can be determined, and therefore the $c \bar{c}$ content inferred. A small contribution to the sample of tagged events is also expected from gluon splitting processes. In this analysis these events are not subtracted from the sample, but will be considered later as a source for a systematic error. Finally for each event a probability is calculated whether this event is $a b \bar{b}$ or a $c \bar{c}$ event. These probabilities are used in the asymmetry fit to separate primary charm and bottom events.

In this section the details and the techniques used to derive the event probabilities are presented. The description starts with a general introduction into the formalism used. Next 
the treatment of background is discussed, and the charm and bottom fractions in the sample are determined. Finally all information is combined in the calculation of the event probabilities.

\subsection{Definition of the Event Probabilities}

The dominant contributions to the sample of events with tagged D mesons come from decays of primary $b$ or $c$ quarks. Only a small fraction of the events is expected to originate from the splitting of a gluon into a pair of charm quarks. For the sake of simplicity this contribution is neglected in the following discussion.

The event probabilities introduced above are calculated individually for each event from lifetime information. They are obtained for two jets in the event, firstly the jet containing the reconstructed $D$ meson, referred to as the $D$ jet in the following, and secondly the jet with the highest energy not containing the $\mathrm{D}$ meson candidate, called the secondary jet. They are calculated, as functions of the $\mathrm{D}$ meson scaled energy, $x=x_{\mathrm{D}}$, and the decay length significance, $\delta$, of the secondary vertex in each jet, by

$$
w_{j}\left(x, \delta^{\mathrm{D}}, \delta^{\mathrm{sec}}\right)=\frac{p_{j}(x) \cdot L_{j}^{\mathrm{D}}\left(x, \delta^{\mathrm{D}}\right) \cdot L_{j}^{\mathrm{sec}}\left(x, \delta^{\mathrm{sec}}\right)}{\sum_{l=\mathrm{bgd}, \mathrm{c}, \mathrm{b}} p_{l}(x) L_{l}^{\mathrm{D}}\left(x, \delta^{\mathrm{D}}\right) L_{l}^{\mathrm{sec}}\left(x, \delta^{\mathrm{sec}}\right)} \quad, j=\mathrm{bgd}, \mathrm{b}, \mathrm{c} .
$$

The functions $L_{j}^{\mathrm{D}}\left(x, \delta^{\mathrm{D}}\right)$ and $L_{j}^{\mathrm{sec}}\left(x, \delta^{\text {sec }}\right)$ are the probability density functions for the $\mathrm{D}$ jet and the secondary jet for the different samples, $j=\mathrm{bgd}, \mathrm{b}, \mathrm{c}$, calculated from the decay length significance distributions. The functions $L_{\text {bgd }}$ are derived from data, as discussed in section 5.2, while the bottom and charm distributions are taken from Monte Carlo simulation. The functions $p_{j}(x)=N_{j}(x) / N_{\text {cand }}(x)$ are the total fractions of the events from the different sources in the candidate sample. These charm and bottom fractions are calculated from lifetime information and jet shape properties as described in section 5.3, in bins of $x_{\mathrm{D}}$, and separately for each channel, from the probability density functions $L_{j}^{\mathrm{D}}\left(x, \delta^{\mathrm{D}}\right)$ and $L_{j}^{\mathrm{sec}}\left(x, \delta^{\text {sec }}\right)$ and also using the output from an artificial neural network analysis based on jet shape information [11].

\subsection{Treatment of Background}

The method used to determine the level of combinatorial background in the sample has been described in section 4.4. For the purpose of flavour separation not only has the absolute level of background to be known, but also the dependence of the background on the separation variable, that is, on decay length significance or jet shape variables.

Data are used as far as possible to determine the background shapes. In the lifetime analysis an estimator is used which is constructed from a $\mathrm{D}^{*+}$ background event tagged by the reflected pion technique discussed in section 4.4. To increase the number of candidates an extended window in the mass difference $\Delta M$ is used. Secondary vertices are searched for in these events and the resulting decay length significance distributions are used to describe the expected background shape as a function of the decay length significance. In the $\mathrm{D}^{0}$ and $\mathrm{D}^{+}$channels the decay length significance background distributions are calculated in sidebands of $M_{\mathrm{D}}$, above (for the $\mathrm{D}^{0}$ and the $\mathrm{D}^{+}$) and below (for the $\mathrm{D}^{+}$) the mass of the expected signal. The region below the $\mathrm{D}^{0}$ mass cannot be used since the decay $\mathrm{D}^{0} \rightarrow \mathrm{K}^{-} \pi^{+} \pi^{0}$ contributes there. Details of the selection of the samples are given in table 4 . 


\begin{tabular}{|c|c|c|c|c|c|}
\hline \multirow[b]{2}{*}{ channel } & \multirow[b]{2}{*}{ variable } & \multicolumn{2}{|c|}{ decay length significance } & \multicolumn{2}{|c|}{ jet shape } \\
\hline & & range & estimator & range & estimator \\
\hline $\begin{array}{l}3 \text { prong } \\
\text { satellite }\end{array}$ & $\Delta M / \mathrm{GeV}$ & $0.140-0.200$ & $\mathrm{R}$ & $0.180-0.200$ & Q \\
\hline 5 prong & & & & & \\
\hline semileptonic & $\Delta M / \mathrm{GeV}$ & $0.140-0.220$ & $\mathrm{R}$ & $0.190-0.220$ & Q \\
\hline $\mathrm{D}^{0}$ & $M_{\mathrm{D}^{0}} / \mathrm{GeV}$ & $1.95-2.50$ & $\mathrm{~S}$ & - & - \\
\hline $\mathrm{D}^{+}$ & $M_{\mathrm{D}^{+}} / \mathrm{GeV}$ & 1.75-1.81,1.93-1.99 & $\mathrm{S}$ & - & - \\
\hline
\end{tabular}

Table 4: Different background estimators used in the seven D meson channels. Shown is the variable in which the background is selected, the range over which events are accepted, and the type of estimator used. For the latter, $\mathrm{S}$ corresponds to the correct charge selection, in the mass range indicated, $\mathrm{Q}$ to the wrong charge one, and $\mathrm{R}$ to the reflected pion sample. Estimators are listed for the decay length significance and the jet shape analysis.

The background sample used in the jet shape analysis is based upon a wrong charge technique, where a $D^{0}$ candidate is reconstructed with an unphysical charge of \pm 2 (e.g. a $\mathrm{K}^{+} \pi^{+}$for the 3 prong channel) by combining tracks appropriately. This is done in sidebands of the $\Delta M$ distribution. The reflected pion estimator has been shown in [11] to bias the jet shapes in the secondary jet significantly and therefore is not used. The ranges for the sidebands are given in table 4 .

Using data estimators for the backgrounds has the advantage that small contributions from partially reconstructed D decays, which are present in all sidebands used, are also present in the background estimator. They therefore do not bias the flavour separation procedure.

The distributions obtained for the decay length significance and the jet shape analysis are used to calculate the probability functions for the background, $L_{\mathrm{bgd}}^{\mathrm{D} \text {,sec }}$. Monte Carlo studies show that the decay length significance and jet shape analysis output distributions expected for true background events are well reproduced by the distributions obtained in the background tagged samples.

\subsection{Determination of the Charm and Bottom Fractions}

The determination of the total bottom and charm fractions $p_{\mathrm{b}}$ and $p_{\mathrm{c}}$ is presented in this section. These fractions are calculated for each channel, separately for each bin of $x_{\mathrm{D}}$.

In the technique adopted, the bottom fraction in the sample of background subtracted events is determined by counting the number of bottom tags present. If $f_{\mathrm{b}}$ is the fraction of $\mathrm{b} \overline{\mathrm{b}}$ events in the sample, and if $N_{\mathrm{b}-\text { tag }}$ events are tagged by the bottom tagging technique, $f_{\mathrm{b}}$ can be derived from

$$
\frac{N_{\mathrm{b}-\text { tag }}}{N_{\text {cand }}}=f_{\mathrm{b}} \mathcal{P}_{\mathrm{b}}+\left(1-f_{\mathrm{b}}\right) \mathcal{P}_{\mathbf{c}}
$$

if the tagging probabilities $\mathcal{P}_{\mathrm{b}}$ and $\mathcal{P}_{\mathrm{c}}$ are known.

The two bottom tagging techniques used are the measurement of the decay length significance and the inclusive jet shape variables which were already mentioned above. In the first 
method hadronic decays of the $\mathrm{Z}^{0}$ into bottom quarks are tagged by taking advantage of the relatively long lifetimes of bottom-flavoured hadrons compared to light-flavoured ones. The decay length significance is measured for the $\mathrm{D}$ candidate and for the background sample. The tagging probabilities $\mathcal{P}_{\mathrm{b}, \mathrm{c}}$ are derived from the decay length significance distributions for c $\overline{\mathrm{c}}$ and $\mathrm{b} \overline{\mathrm{b}}$ events, which are taken from Monte Carlo simulation. An event is tagged as a $\mathrm{b} \overline{\mathrm{b}}$ event if the decay length significance is larger than 8 . The procedure is repeated for a value of $\delta=2,4$ and 6 , and the results are combined taking the statistical correlations of the samples into account. This measurement is performed in both the $\mathrm{D}$ jet and the secondary jet.

The second method uses the shapes of jets, which are expected to be significantly different for bottom and charm jets. The shapes are measured by a set of seven jet shape variables, containing combinations of the momenta of the particles in the jets, where both transverse and longitudinal components are used. The shape variables are only calculated for the secondary jet in the event, which does not contain the $\mathrm{D}$ meson candidate, since the $\mathrm{D}$ meson candidate does introduce a significant bias into the $\mathrm{D}$ jet. These variables are used as input to an artificial neural network which has been trained using data and Monte Carlo for b/c separation. A detailed description of the variables and the method may be found in [11]. The tagging probabilities $\mathcal{P}_{\mathrm{b}}$ have been calculated in [11] from data, using samples enriched in bottom decays by the presence of a high momentum lepton. The tagging probabilities for $c \bar{c}$ events, $\mathcal{P}_{\mathbf{c}}$, have been determined using Monte Carlo simulation. They are calculated for four ranges of the output of the neural network. The number of events found in each range is determined, and the total $b \bar{b}$ fraction calculated. The network was developed for the analysis of $\mathrm{D}^{*+}$ decays and is applied to the $\mathrm{D}^{*+}$ sample only.

The results of this part of the analysis are the charm fractions in the background subtracted event sample, $f_{\mathrm{c}}\left(x_{\mathrm{D}}\right)_{\text {channel}}$, determined as a function of the scaled energy for each channel separately. The charm fraction in the total sample of events including the background is derived from this through the relation $p_{\mathrm{b}, \mathrm{c}}=f_{\mathrm{b}, \mathrm{c}}\left(1-N_{\mathrm{bgd}} / N_{\text {cand }}\right)$. In table 5 the charm fractions are given combined into two bins of $x_{\mathrm{D}}$, for each channel. The systematic errors shown in the table are discussed in detail later. The decay length significance and the neural net output distributions are shown in figure 3 for the background subtracted candidate sample. The expected distributions for $c \bar{c}$ events are superimposed.

\subsection{Calculation of the Event Probabilities}

The measurement of the decay length significance for each event is used to determine the probabilities defined in eq. 5 . In addition, the charm and bottom fractions $p_{\mathbf{c}}$ and $p_{\mathrm{b}}$, derived with b tagging methods as described in section 5.3, are needed to calculate the event probabilities. In nearly all events at least one vertex is reconstructed, either in the $D$ jet or in the secondary jet, from which a valid event probability can be calculated. If no vertex is reconstructed in one of the two jets considered, an event probability is still calculated if the reconstruction was successful in the other jet. If the vertex reconstruction failed in both jets considered, the event based probabilities are replaced by the mean flavour fraction, determined from the $p_{j}(x)$ only. The fraction of such events is less than $1 \%$ of the total sample. 


\begin{tabular}{|c|c|c|}
\hline channel & $0.2<x_{\mathrm{D}}<0.4$ & $x_{\mathrm{D}}>0.4$ \\
\hline 3 prong & $0.258 \pm 0.020 \pm 0.027$ & $0.720 \pm 0.017 \pm 0.025$ \\
\hline satellite & $0.358 \pm 0.019 \pm 0.035$ & $0.797 \pm 0.015 \pm 0.025$ \\
\hline$n g$ & - & $0.841 \pm 0.017 \pm 0.032$ \\
\hline electron & $0.433 \pm 0.038 \pm 0.030$ & $0.876 \pm 0.029 \pm 0.024$ \\
\hline \multirow[t]{2}{*}{ muon } & $0.306 \pm 0.036 \pm 0.028$ & $0.867 \pm 0.029 \pm 0.026$ \\
\hline & $0.3<x_{\mathrm{D}}<0.5$ & $x_{\mathrm{D}}>0.5$ \\
\hline $\mathrm{D}^{0}$ & $0.369 \pm 0.034 \pm 0.099$ & $0.787 \pm 0.026 \pm 0.108$ \\
\hline $\mathrm{D}^{+}$ & $0.317 \pm 0.060 \pm 0.130$ & $0.810 \pm 0.035 \pm 0.077$ \\
\hline
\end{tabular}

Table 5: List of charm fractions, $f_{c}$, in the different channels with their statistical and systematic errors. Results are shown both for two ranges of $x_{\mathrm{D}^{*+}}$ to illustrate the increasing charm purity with increasing $x_{\mathrm{D}}$. Note that the $x_{\mathrm{D}}$ used is the one calculated from the observed tracks, and the same $x_{\mathrm{D}}$ could correspond to a different true scaled energy of the $\mathrm{D}$ meson in different channels.

\section{Determination of the Asymmetries}

In this section the method used to determine the asymmetries is presented. While the main thrust of the analysis is the measurement of the charm forward-backward asymmetry, a simultaneous fit is done for both bottom and charm. At the same time the method presented allows the measurement of the effective mixing of $B$ mesons applicable to this sample of events. The results of the fit are discussed in section 6.4.

\subsection{The Likelihood Function}

The forward-backward asymmetry is defined as

$$
A_{\mathrm{FB}}=\frac{\int_{0}^{1} \frac{\mathrm{d} \sigma}{\mathrm{d} y} \mathrm{~d} y-\int_{-1}^{0} \frac{\mathrm{d} \sigma}{\mathrm{d} y} \mathrm{~d} y}{\int_{0}^{1} \frac{\mathrm{d} \sigma}{\mathrm{d} y} \mathrm{~d} y+\int_{-1}^{0} \frac{\mathrm{d} \sigma}{\mathrm{d} y} \mathrm{~d} y}
$$

where $y$ is the cosine of the polar angle of the outgoing fermion direction with respect to that of the incoming fermion. In events containing a $\mathrm{D}$ meson, the direction $y$ is approximately described by $y=q \cos \left(\theta_{\text {thrust }}\right)$, where $q$ is the sign of the charge of the $\mathrm{D}$ meson ${ }^{4}$ and $\theta_{\text {thrust }}$ is the angle of the thrust axis with respect to the electron beam. The orientation of the thrust axis is chosen such that the scalar product of the thrust axis with the $\mathrm{D}$ direction is positive.

The asymmetry is determined in an unbinned log-likelihood fit to the $y$ distribution. The likelihood function has the form (c.f. eq. 1)

$$
\log \mathcal{L}=\sum_{i} \log \left(1+y_{i}^{2}+\frac{8}{3} A_{\mathrm{FB}}^{\mathrm{obs}} y_{i}\right)-\sum_{i} \log N_{\text {norm }}^{i} .
$$

\footnotetext{
${ }^{4}$ The charge of the D meson is defined as the sign of the charge of the charm quark in the quark model for the meson.
} 
The sum runs over all candidates $i$ considered. Since the normalisation does not depend on the asymmetry itself, provided the acceptance is symmetric in $y$, it does not contribute to the maximisation of the likelihood, and is dropped from the further discussion.

The total observed asymmetry has contributions from bottom, charm and background events, resulting in a likelihood for event $i$ of

$$
\mathcal{L}_{i}=1+y_{i}^{2}+\frac{8}{3}\left[w_{\mathrm{b}}^{i}\left(1-2 C_{\mathrm{D}}^{i}(\delta)\right) A_{\mathrm{FB}}^{\mathrm{b}}+w_{\mathrm{c}}^{i} A_{\mathrm{FB}}^{\mathrm{c}}+w_{\mathrm{bgd}}^{i} A_{\mathrm{FB}}^{\mathrm{bgd}}\right] y_{i} .
$$

Here $w_{\mathrm{bgd}, \mathrm{b}, \mathrm{c}}$ are the probabilities that a $\mathrm{D}$ meson originates from the source indicated and $A_{\mathrm{FB}}^{\mathrm{bgd}}$ allows for a possible asymmetry in the background (see section 6.2). Mixing and non-prompt production of $\mathrm{D}$ mesons can change the charge correlation between the primary quark and the detected $\mathrm{D}$ meson in the bottom sample. These effects are collectively described by the charge correlation factor $C_{\mathrm{D}}^{i}(\delta)$ and described in section 6.3 .

\subsection{Background Asymmetry}

Background in the sample is expected to have no asymmetry if the sign of the charge reconstructed for the background, $q_{\mathrm{cand}}$, is uncorrelated to the sign of the charge of the primary quark, $q_{\text {prim }}$, in the event. If a correlation exists between $q_{\text {cand }}$ and $q_{\text {prim }}$ for a significant fraction of events, the background displays a non-zero asymmetry. Monte Carlo studies show that such correlations are expected to be present at a significant level in the different channels. These correlations come mostly from partially reconstructed or misreconstructed D meson decays in the background samples. These studies also indicate that the amount of correlation does not depend on $\Delta M, M_{\mathrm{D}^{0}}$ or $M_{\mathrm{D}^{+}}$, allowing it to be measured in sidebands in $\Delta M, M_{\mathrm{D}^{0}}$ and $M_{\mathrm{D}^{+}}$, respectively. From the simulation it is found that typical charge correlations are of the order of a few percent in the non-leptonic D channels, and around $10 \%$ in the semileptonic channels. Together with the expected asymmetries for $q \bar{q}$ events, which are below $10 \%$, this translates into background asymmetries of typically less than $1 \%$. Nevertheless the fit is extended to determine simultaneously the background asymmetries. This is done by including the sideband regions in the fit. Since the statistical significance of the events found in the sidebands is as good as, or better than, that of the candidates in the signal region, this essentially constrains the background asymmetries to their values in the sideband, and allows them to be included in the fit without loss of precision for the $c \bar{c}$ and $b \bar{b}$ asymmetry determination. The Monte Carlo simulation indicates that the sidebands describe the charge asymmetries to better than $0.1 \%$, with no significant systematic effects visible. The ranges of the sidebands are the same as those summarised in table 4 , but only candidates with the correct charge combination of candidate tracks are used in the estimation.

\subsection{Determination of $C_{D}$ in $b \bar{b}$ Events}

The correlation between the charge of the detected $\mathrm{D}$ meson and the charge of the produced quark in $b \bar{b}$ events can be changed by two basic processes: Mixing in the neutral B sector and the production of a D meson in $\mathrm{b} \rightarrow(\bar{c} s) c$, where the reconstructed $\mathrm{D}$ meson contains the charm quark produced from the virtual $\mathrm{W}$. The amount of charge correlation from $\mathrm{B}^{0}-\overline{\mathrm{B}^{0}}$ mixing depends on the proper decay time of the $\mathrm{D}$ meson, whereas the correlation introduced from the second process does not. Small effects are also expected from $\mathrm{B}_{\mathrm{s}}^{0}-\overline{\mathrm{B}_{\mathrm{s}}^{0}}$ mixing. 
Since in this analysis the lifetime information in the D hemisphere is used to help separate $\mathrm{b} \overline{\mathrm{b}}$ and $\mathrm{c} \overline{\mathrm{c}}$ events, $C_{\mathrm{D}}$ has to be known as a function of the decay length significance. Events with a large measured decay length significance are likely to be $b$ events, and therefore will enter in the fit with a large probability to be a bottom event. Such events are also more likely to have undergone mixing, and have consequently a different charge correlation between the primary $\mathrm{b}$ and the tagged $\mathrm{D}$ meson. Therefore the appropriate charge correlation factor $C_{\mathrm{D}}$ for a certain measured decay length significance has to be used in the likelihood fit.

The charge correlation in $b \bar{b}$ events is directly measured in the sample of $\mathrm{D}^{*+}$ mesons used for the asymmetry fit. For this part of the analysis only $\mathrm{D}^{*+}$ mesons are used because of their lower background level compared to the $\mathrm{D}^{0}$ and $\mathrm{D}^{+}$channels. The charge of the primary quark is measured by comparing charges in both hemispheres of the event, where the charge information from the reconstructed $\mathrm{D}^{*+}$ meson is used in one hemisphere, and a jet charge technique in the other hemisphere. The charge correlation is calculated from the ratio of events with opposite charges in both hemispheres to the total number of events. It is determined as a function of the decay length significance of the $\mathrm{D}$ candidate and parametrised by a function of the form $[16]$

$$
C_{\mathrm{D}}^{\prime}(\delta)=a^{\prime}+b^{\prime} \sin ^{2}(\nu \delta),
$$

where $a^{\prime}, b^{\prime}$ and $\nu$ are determined in the fit. The charge correlation function $C_{\mathrm{D}}(\delta)$ with parameters $a$ and $b$ is calculated from $C_{\mathrm{D}}^{\prime}$ in eq. 10 by correcting for the probability for correct jet charge identification.

Eq. 10 also has contributions from the background and from c $\bar{c}$ events. The charm background is suppressed by considering only events with $x_{\mathrm{D}^{*+}}<0.4$ for this part of the analysis. In the following the details of the analysis and the treatment of the different backgrounds are discussed in detail.

\subsubsection{Measurement of Jet Charge Identification Probabilities}

The jet charge used is defined as a momentum weighted sum over the charges of the tracks in the hemisphere considered [17]:

$$
Q_{j e t}=\frac{\sum_{i}^{N}\left|p_{\|}^{i}\right|^{\kappa} q_{i}}{\sum_{i}^{N}\left|p_{\|}^{i}\right|^{\kappa}},
$$

where the sum runs over the $N$ charged tracks of the hemisphere, $p_{\|_{i}}$ is the momentum component of the track $i$ along the thrust axis, $q_{i}$ is the charge of track $i$ and $\kappa$ is a parameter which defines a momentum weighting for each particle. The weight factor optimal for the identification of the primary quark charge in the event has been determined to be $\kappa=0.3[18]$. The sign of the jet charge $Q_{j e t}$ is used to reconstruct the charge of the hemisphere.

The jet charge identification probability $\eta_{j}$ for the different flavours $j$ is defined as the fraction of events where the charge of the primary quark is correctly identified. The jet charge identification probabilities for charm and bottom events are measured in data with samples of events enriched in each flavour.

A highly enriched sample of bottom decays is selected by identifying leptons with high momentum, $p$, and high momentum transverse to the jet direction, $p_{t}$, opposite to the hemisphere where the jet charge is determined. The b purity of the lepton sample is $(91.2 \pm 2.0) \%$ [14] for the selection cuts of $p>2 \mathrm{GeV}$ and $p_{t}>1.3 \mathrm{GeV}$ for electron candidates, and $p>3 \mathrm{GeV}$ and 
$p_{t}>1.5 \mathrm{GeV}$ for muon candidates. From this the jet charge identification probability in $\mathrm{b} \overline{\mathrm{b}}$ events is determined to be $\eta_{\mathrm{b}}=0.642 \pm 0.003 \pm 0.013$ where the first error is statistical and the second systematic. The systematic error is dominated by the errors on the bottom purity of the sample and the charm background.

Decays of the $\mathrm{Z}^{0}$ into $\mathrm{c} \overline{\mathrm{c}}$ pairs are selected by requiring a reconstructed $\mathrm{D}^{*+}$ meson in one of the five channels in the opposite hemisphere with a large scaled energy $x_{\mathrm{D}^{*+}}>0.7$. This sample is found to have a signal purity of $(72.4 \pm 1.1) \%$ and a charm purity of the $\mathrm{D}^{*+}$ signal events of $(96.9 \pm 0.4 \pm 2.0) \%$ (see section 5.3). From this the jet charge identification probability in $c \bar{c}$ events is calculated to be $\eta_{\mathrm{c}}=0.699 \pm 0.010 \pm 0.012$, where the first error is statistical and the second systematic. Possible charge correlations in the background are corrected for by repeating the analysis in the sidebands of the $\Delta M$ distributions, analogous to the method described in section 6.2. The main systematic error is due to the charm purity of the sample, and possible differences between the charge correlation of the background and the sidebands.

\subsubsection{Determination of $C_{\mathrm{D}}(\delta)$}

The data sample of identified D mesons is divided into two samples, a like sign sample, where the sign of the reconstructed D meson is equal to that of the jet charge, and an unlike sign sample. If the charge of the primary quark is correctly tagged by the jet charge, mesons produced in $\mathrm{b} \rightarrow(\overline{\mathrm{c} s}) \mathrm{c}$ decays, and those produced in $\mathrm{B}$ decays where the $\mathrm{B}$ meson had mixed before decaying, would contribute to the unlike sign sample. Unmixed events would contribute to the like sign sample.

In the sample of tagged unlike sign events additional contributions are present from a variety of background processes. The combinatorial background has been measured and is given in table 3 . The remaining charm contribution in this sample has been measured as described in section 5.3 and found to be $(34.5 \pm 0.8 \pm 2.5) \%$.

These two background sources are subtracted from the sample. Firstly the combinatorial background is subtracted as a function of the decay length significance. The shape of this background is estimated using the same sideband tagged events as the background estimator as discussed in section 6.2. Secondly, the charm background is subtracted with the decay length significance distributions taken from Monte Carlo simulation, after taking the jet charge identification probability for charm into account. The resulting decay length significance distributions contain only $b \bar{b}$ events. They are derived for both unlike sign events and for all candidates. The total fraction of unlike sign events in the $b$ sample at decay length significance $\delta$ can be written as :

$$
\frac{N_{\mathrm{b}}^{\text {unlike }}(\delta)}{N_{\mathrm{b}}^{\mathrm{all}}(\delta)}=\eta_{\mathrm{b}} r^{+-}(\delta)+\left(1-\eta_{\mathrm{b}}\right)\left(1-r^{+-}(\delta)\right)=C_{\mathrm{D}}^{\prime}(\delta)
$$

Here $\eta_{b}$ denotes the jet charge identification probability, and $r^{+-}$the fraction of $\mathrm{D}^{*+}$ mesons with opposite charge correlation, i.e. where the $\mathrm{B}$ meson had undergone mixing or the $\mathrm{D}^{*+}$ meson has been produced with the wrong sign in a bottom decay $\mathrm{b} \rightarrow(\overline{\mathrm{c} s}) \mathrm{c}$.

The ratio of unlike sign to all events is fitted with the function $C_{\mathrm{D}}^{\prime}(\delta)$ in eq. 10 as a function of the decay length significance. Free parameters in the fit are the offset $a^{\prime}$, the amplitude $b^{\prime}$ and the frequency $\nu$. 
The variables $a^{\prime}$ and $b^{\prime}$ depend on the fraction of $\mathrm{D}^{*+}$ mesons originating from $\mathrm{b} \rightarrow(\overline{\mathrm{cs}}) \mathrm{c}$ decays, $\mathrm{B}_{\mathrm{s}}^{0}$ mesons and charged $\mathrm{B}$ mesons. In addition experimental resolution effects both from the jet charge reconstruction and from the decay length significance reconstruction influence these parameters. The measured parameters $a^{\prime}$ and $b^{\prime}$ are corrected for the effects of the jet charge identification efficiencies according to

$$
\begin{aligned}
a & =\frac{a^{\prime}+\eta_{\mathrm{b}}-1}{2 \eta_{\mathrm{b}}-1} \\
b & =\frac{b^{\prime}}{2 \eta_{\mathrm{b}}-1} .
\end{aligned}
$$

The corrected parameters together with the measured frequency $\nu$ define the function $C_{\mathrm{D}}(\delta)$ in the asymmetry fit. Care has to be taken in interpreting the results of the fit in terms of $\mathrm{B}^{0}-\overline{\mathrm{B}^{0}}$ mixing. In $[16]$ eq. 10 is used as a function of the proper time of the $\mathrm{B}$ hadron in the event, while in this analysis the decay length significance is used. Although this does change the interpretation of the results, Monte Carlo studies show that eq. 10 still provides a good description of the decay length significance dependence of the charge correlation.

The distribution of the ratio of unlike sign to all events as a function of the decay length significance after background and charm subtraction is shown in figure 4. The dependence of this ratio on the decay length significance is clearly visible. The result of the fit using eq. 10 is superimposed on the plot.

To compare the result of the fit with other related measurements of the mixing in the neutral B system, the effective mixing parameter $\chi_{\text {eff }}$ is derived from the fitted parameters $a, b$ and $\nu$. This effective mixing parameter takes into account the fact that $\mathrm{D}^{*+}$ samples are produced in a mixture of $\mathrm{B}^{0}, \mathrm{~B}^{+}, \mathrm{B}_{\mathrm{s}}^{0}$ and $\mathrm{b} \rightarrow(\overline{\mathrm{c} s}) \mathrm{c}$ decays. The effective mixing is determined from the individual mixing in each component and the relative importance of the different contributions. It is found to be $\chi_{\mathrm{eff}}=(19.1 \pm 8.3) \%$. This should be compared to the same quantity as estimated from other measurements (see [10] for a derivation) of $\chi_{\mathrm{eff}}=(17.8 \pm 4.3) \%$, in good agreement with the direct determination. Note, however, that in this asymmetry analysis no knowledge of the actual physical mixing parameter $\chi_{\mathrm{eff}}$ is needed.

The fractions of $\mathrm{D}^{0}$ or $\mathrm{D}^{+}$mesons produced in $\mathrm{B}^{0}$ and $\mathrm{B}_{\mathrm{s}}^{0}$ meson decays are different from that of $\mathrm{D}^{*+}$ mesons. This is mostly due to excited charm states and their decays into $\mathrm{D}^{0}$ and $\mathrm{D}^{+}$mesons. These differences have been estimated in [15] and the factors $a$ and $b$ recalculated. The decay length significance dependence of the charge correlation $C_{\mathrm{D}}(\delta)$ is assumed to be the same as the $\mathrm{D}^{*+}$ mesons. From this the effective mixing for the $\mathrm{D}^{0}$ tagged sample is estimated to be $\chi_{\mathrm{eff}}=(8.0 \pm 4.4) \%$ and for the $\mathrm{D}^{+}$sample $\chi_{\mathrm{eff}}=(19.4 \pm 4.2) \%$.

\subsection{Results of the Fit}

The samples collected on, below and above the peak of the $\mathrm{Z}^{0}$ resonance have been fitted to determine $A_{\mathrm{FB}}^{\mathrm{c}}$ and $A_{\mathrm{FB}}^{\mathrm{b}}$. The final probability density function used to construct the likelihood is given by equation 9 . The bottom asymmetry, the charm asymmetry and the background asymmetries are free parameters in the fit. The latter are allowed to vary independently in each of the seven channels considered. It should be noted, however, that the background asymmetries are nearly completely determined from the sidebands, and are very much decoupled from the charm and bottom asymmetries. Typical correlations between the background asymmetries and the charm or bottom asymmetries are below $10 \%$. 


$$
\begin{aligned}
A_{\mathrm{FB}}^{\mathrm{c}}=0.039 \pm 0.051 & A_{\mathrm{FB}}^{\mathrm{b}}=-0.086 \pm 0.108 & \left\langle E_{c m}\right\rangle & =89.45 \mathrm{GeV} \\
A_{\mathrm{FB}}^{\mathrm{c}}=0.063 \pm 0.012 & A_{\mathrm{FB}}^{\mathrm{b}}=0.094 \pm 0.027 & \left\langle E_{c m}\right\rangle & =91.22 \mathrm{GeV} \\
A_{\mathrm{FB}}^{\mathrm{c}}=0.158 \pm 0.041 & A_{\mathrm{FB}}^{\mathrm{b}}=-0.021 \pm 0.090 & \left\langle E_{c m}\right\rangle & =93.00 \mathrm{GeV}
\end{aligned}
$$

with a statistical correlation coefficient between the bottom and charm asymmetry of -0.281 on-peak $(-0.272$ and -0.273 for the low and high energy off-peak points, respectively). The centre-of-mass energies indicated are event weighted averages of the appropriate off-peak and on-peak points [19].

The result of the fit for the on-peak data is illustrated in figure 5 , where the differential asymmetry, $\Delta A_{\mathrm{FB}}=(n(y)-n(-y)) /(n(y)+n(-y))$ is shown as a function of $y$, and $n( \pm y)$ are the number of candidates in the forward (backward) $y$ hemisphere. The results are plotted as a function of the centre of mass energy in figure 6. Also shown is the standard model prediction evaluated using the ZFITTER model [20] for a top mass of $180 \mathrm{GeV}$ [15] and a Higgs mass of $300 \mathrm{GeV}$. The correlation between $A_{\mathrm{FB}}^{\mathrm{c}}$ and $A_{\mathrm{FB}}^{\mathrm{b}}$ is illustrated in figure 7, together with the predictions of the standard model shown for the asymmetries on the peak of the $Z^{0}$ resonance.

The fit has been repeated with the bottom asymmetry set to its standard model expectation obtained for a Higgs mass of $300 \mathrm{GeV}$ and a top mass of $180 \mathrm{GeV}$. The results of the fits are

$$
\begin{array}{lll}
A_{\mathrm{FB}}^{\mathrm{c}}=0.020 \pm 0.049 & \left\langle E_{c m}\right\rangle=89.45 \mathrm{GeV} \\
A_{\mathrm{FB}}^{\mathrm{c}}=0.064 \pm 0.012 & \left\langle E_{c m}\right\rangle=91.22 \mathrm{GeV} \\
A_{\mathrm{FB}}^{\mathrm{c}}=0.139 \pm 0.039 & \left\langle E_{c m}\right\rangle=93.00 \mathrm{GeV}
\end{array}
$$

in good agreement with the above two parameter fit.

\section{Cross Checks and Systematic Errors}

The stability of the fit procedure has been checked by repeating the analysis individually for each of the seven channels and comparing the results. Consistent results are obtained.

Two groups of systematic errors affect the analysis. The first group is related to errors specific to this analysis and the determination of the asymmetry. The second group concerns errors related to the separation of the sample into its bottom and charm components. A more detailed description of the errors relating to the determination of the charm fraction can be found in [11].

The following sources relevant for the asymmetry analysis have been considered:

- Experimental acceptance: One of the advantages of the likelihood fit is that it is independent of the experimental acceptances. This is only true if the ratio between signal and background acceptances is constant over the different $\cos \theta$ regions for which they have been determined. The influence of this assumption has been investigated by repeating the fit with signal and background determined in only one bin of $\cos \theta$. Possible deviations from this assumption could be caused by a variation of the mass resolution with $\cos \theta$, which might affect signal and background differently. The effect is found to be small. 


\begin{tabular}{|c|c|c|c|c|c|c|}
\hline \multirow{2}{*}{$\begin{array}{l}\left\langle E_{c m}\right\rangle \\
\text { Systematic error source }\end{array}$} & \multicolumn{2}{|c|}{$89.45 \mathrm{GeV}$} & \multicolumn{2}{|c|}{$91.22 \mathrm{GeV}$} & \multicolumn{2}{|c|}{$93.00 \mathrm{GeV}$} \\
\hline & $\Delta\left(A_{\mathrm{FB}}^{\mathrm{c}}\right)$ & $\Delta\left(A_{\mathrm{FB}}^{\mathrm{b}}\right)$ & $\Delta\left(A_{\mathrm{FB}}^{\mathrm{c}}\right)$ & $\Delta\left(A_{\mathrm{FB}}^{\mathrm{b}}\right)$ & $\Delta\left(A_{\mathrm{FB}}^{\mathrm{c}}\right)$ & $\Delta\left(A_{\mathrm{FB}}^{\mathrm{b}}\right)$ \\
\hline General errors & & & & & & \\
\hline $\begin{array}{l}\text { same acceptance for } \\
\text { background and signal }\end{array}$ & 0.0009 & 0.0028 & 0.0009 & 0.0028 & 0.0009 & 0.0028 \\
\hline Background level & 0.0038 & 0.0101 & 0.0014 & 0.0062 & 0.0038 & 0.0113 \\
\hline Charge correlation & 0.0010 & 0.0164 & 0.0010 & 0.0186 & 0.0028 & 0.0037 \\
\hline $\mathrm{g} \rightarrow \mathrm{c} \overline{\mathrm{c}}, \mathrm{b} \overline{\mathrm{b}}$ & +0.0002 & -0.0034 & +0.0020 & +0.0034 & +0.0050 & -0.0001 \\
\hline Background asymmetry & 0.0010 & 0.0023 & 0.0010 & 0.0023 & 0.0010 & 0.0023 \\
\hline $\begin{array}{l}x_{\mathrm{D}} \text { dependence of } \\
\text { background asymmetry }\end{array}$ & 0.0002 & 0.0001 & 0.0003 & 0.0001 & 0.0008 & 0.0001 \\
\hline$b$-c separation errors & & & & & & \\
\hline Monte Carlo statistics & 0.0042 & 0.0124 & 0.0005 & 0.0010 & 0.0022 & 0.0130 \\
\hline Background estimator & 0.0018 & 0.0055 & 0.0018 & 0.0055 & 0.0018 & 0.0055 \\
\hline Charged mult. in b jets & -0.0020 & +0.0095 & -0.0016 & +0.0017 & -0.0040 & +0.0100 \\
\hline Charged mult. in $\mathrm{c}$ jets & +0.0015 & -0.0065 & +0.0011 & -0.0012 & +0.0040 & +0.0100 \\
\hline Charm modelling & 0.0030 & 0.0054 & 0.0030 & 0.0054 & 0.0030 & 0.0054 \\
\hline Detector response & 0.0015 & 0.0045 & 0.0005 & 0.0006 & 0.0008 & 0.0022 \\
\hline Detector resolution & 0.0011 & 0.0006 & 0.0011 & 0.0006 & 0.0011 & 0.0006 \\
\hline Heavy flavour frag. & 0.0005 & 0.0015 & 0.0003 & 0.0005 & 0.0007 & 0.0015 \\
\hline Bottom hadron lifetime & -0.0030 & +0.0075 & -0.0015 & +0.0025 & -0.0021 & +0.0072 \\
\hline Charmed hadron lifetime & +0.0030 & -0.0054 & +0.0012 & -0.0018 & +0.0030 & -0.0054 \\
\hline Total & 0.0087 & 0.0292 & 0.0055 & 0.0220 & 0.0110 & 0.0259 \\
\hline
\end{tabular}

Table 6: List of the systematic errors contributing to the measured asymmetries of the processes $\mathrm{e}^{+} \mathrm{e}^{-} \rightarrow \mathrm{c} \overline{\mathrm{c}}$ and $\mathrm{e}^{+} \mathrm{e}^{-} \rightarrow \mathrm{b} \overline{\mathrm{b}}$, for the three mean centre-of-mass energies investigated. A sign in front of an error indicates the direction of change under a positive change of the variable.

- Asymmetric acceptance: The acceptance of the detector has been studied for signs of possible asymmetries in $q \cos \theta$. No indication of any effect has been found, either in the simulation or in the data.

- Background level: Uncertainties in the background fraction can introduce a bias in the asymmetry. This has been investigated by varying the background fractions within their systematic errors or $\pm 3 \%$, as given in [11].

- Charge correlation: The charge correlation function $C_{\mathrm{D}}(\delta)$ is determined from data. The parameters of the function $C_{\mathrm{D}}(\delta)$ have been varied within their errors as indicated in figure 4 , taken correlations between the parameters into account. Any resulting differences in the asymmetries are quoted as a systematic error.

- Gluon splitting $\mathrm{g} \rightarrow \mathrm{c} \overline{\mathrm{c}}, \mathrm{g} \rightarrow \mathrm{b} \overline{\mathrm{b}}$ : Heavy flavour production in gluon initiated jets will contribute at a low level to the sample of tagged D mesons. First measurements of this contribution have been presented in $[11,21]$, and found to be in agreement with current theoretical predictions. The measured rate of $c \bar{c}$ production from gluon splitting per hadronic event is $n_{\mathbf{g} \rightarrow \mathbf{c}}=(2.38 \pm 0.48) \%$. Systematic errors on the charm and bottom 
asymmetries have been calculated by assuming that all events originating from gluon splitting were assigned by the $\mathrm{b} / \mathrm{c}$ separation to be $c \bar{c}$ events or $\mathrm{b} \overline{\mathrm{b}}$ events, respectively. The measured asymmetries have not been corrected explicitely for the effects of gluon splitting, because the event probabilities do not provide a clear assignment of these events to a specific flavour. By applying the lifetime method to the $\mathrm{D}$ jet, these events will most probably be counted as charm events, whereas the lifetime information in the secondary jet will preferentially assign them to the primary event flavour.

- Background asymmetries: The determination of the background asymmetries depends on the assumption that the sidebands correctly reproduce the charge correlation present in the true background. This assumption has been checked in the simulation. Typical charge correlations are found to be of the order of a few percent, and differences between sideband and true background below $0.1 \%$. The observed differences are used as systematic errors from this source.

- $x_{\mathrm{D}}$ dependence of the background asymmetries: A possible bias in the background asymmetries as a function of $x_{\mathrm{D}}$ has been investigated by splitting the background samples into two bins of $x_{\mathrm{D}}$ and repeating the fit. The difference has been assigned as the systematic error. Its size is also consistent with Monte Carlo studies.

The errors considered in connection with the flavour separation are:

- Monte Carlo statistics: The determination of the tagging probabilities relies partially on Monte Carlo simulation. An error arises owing to the limited statistics of the Monte Carlo available.

- Background estimators: Some differences may exist between the true background and the background estimators used. This has been estimated in Monte Carlo by repeating the fit with the true background.

- Heavy flavour multiplicity: The multiplicity for heavy flavour decays in the Monte Carlo has been varied by reweighting simulated events, corresponding to the current experimental uncertainties of \pm 0.5 tracks in $\mathrm{b} \overline{\mathrm{b}}$ events and \pm 0.2 tracks in $c \bar{c}$ events [22]. The systematic errors of the off-peak measurements are greater because they contain a statistical component.

- Charm modelling: The modelling of the shape of charm jets, of their multiplicities and momentum flow, is another important source of systematic error in the jet shape analysis. This error also includes effects due to possible differences between data and simulation in the decay length distribution of jets originating from charm quark decays, not already covered by the other charm modelling error. This latter part of the error has been calculated by repeating the analysis after determining the charm decay length distribution nearly entirely from data. This is possible, since the decay length significance distribution of the candidates, of the background and of the bottom jets are known. The observed differences in the asymmetries are used as systematic errors.

- Detector response: Non-uniformity of the detector response as a function of $\cos \theta$ gives rise to this error. This error is present only in the jet shape analysis. 
- Detector resolution: The resolution of the track parameters in the Monte Carlo is varied by $\pm 5 \%$.

- Heavy flavour fragmentation: The parameters for the charm and bottom fragmentation function are varied within their experimental limits $[22]$ of $\epsilon_{\mathbf{c}}=0.035 \pm 0.009$ and $\epsilon_{\mathrm{b}}=$ $0.0024_{-0.0006}^{+0.0023}$.

- Bottom hadron lifetime: The B-hadron lifetime has been varied within its current experimental error. For the flavour separation performed in the hemisphere opposite to the $\mathrm{D}$ mesons, the mean lifetime of bottom hadrons as measured at LEP has been varied by $\pm 0.021 \mathrm{ps}$. In the $\mathrm{D}$ hemisphere, the individual $\mathrm{B}$ hadron lifetimes have been varied independently by $\pm \mathbf{0 . 0 7} \mathrm{ps}$ for the $\mathrm{B}^{+}, \pm \mathbf{0 . 0 8} \mathrm{ps}$ for the $\mathrm{B}^{\mathbf{0}}$, and $\pm \mathbf{0 . 1 2} \mathrm{ps}$ for the $\mathrm{B}_{\mathrm{s}}^{0}[15]$.

- Charm lifetime: The lifetime of the weakly decaying charm hadrons $\mathrm{D}^{0}$ and $\mathrm{D}^{+}$has been varied independently by \pm 0.004 ps for the $\mathrm{D}^{0}$, and $\pm \mathbf{0 . 0 1 5}$ ps for the $\mathrm{D}^{+}[15]$.

A list of all systematic errors considered is given in table 6 .

\section{Pole Asymmetries and Interpretation of Results}

The measured asymmetries presented above are corrected for the effects of initial and final state photon and final state gluon radiation following the procedure proposed by the LEP collaborations in $[22,23]$. The corrections are summarised in table 7.

QED corrections are mostly due to initial state radiation. The QCD corrections take into account that the thrust axis is used in these measurements to approximate the quark direction. Photon exchange in addition to $\mathrm{Z}^{0}$ exchange contributes to the signal, and a small correction is needed to correct for the centre-of-mass energy at which LEP operated, which is not exactly at the nominal $\mathrm{Z}^{0}$ mass.

The asymmetry measurements in the three energy ranges are combined into one by extrapolating the off-peak measurements to the peak using the standard model energy dependence. Applying the corrections mentioned above to the measurements of the charm and bottom asymmetries yields

$$
A_{\mathrm{FB}}^{\mathrm{c}, 0}=0.077 \pm 0.013 \quad A_{\mathrm{FB}}^{\mathrm{b}, 0}=0.078 \pm 0.033
$$

where all errors have been combined in quadrature.

The forward-backward pole asymmetry is closely related to the effective weak mixing angle, $\sin ^{2} \theta_{\text {eff }}^{\text {lept }}$, and the weak coupling constants of the vector- and the axial-vector neutral current, $g_{\mathrm{V}}$ and $g_{\mathrm{A}}$. Following equations 2,3 and $4, \sin ^{2} \theta_{\mathrm{W}}^{\text {eff, lept }}$ can be calculated from the charm and bottom pole asymmetries, when the flavour dependence of the radiative corrections to the electroweak couplings is taken into account. This is done by using the program ZFITTER [20]. After combining the results derived from the charm and bottom pole asymmetries, the following value for the weak mixing angle is found:

$$
\sin ^{2} \theta_{\mathrm{W}}^{\text {eff, lept }}=0.2313 \pm 0.0028 .
$$




\begin{tabular}{|l|l|l|}
\hline \hline Source & Shift in $A_{\mathrm{FB}}^{\mathrm{c}}$ & Shift in $A_{\mathrm{FB}}^{\mathrm{b}}$ \\
\hline \hline QED corrections & $+\mathbf{0 . 0 1 0 4}$ & $+\mathbf{0 . 0 0 4 1}$ \\
QCD corrections & $+\mathbf{0 . 0 0 2 3} \pm \mathbf{0 . 0 0 0 7}$ & $+\mathbf{0 . 0 0 3 3} \pm \mathbf{0 . 0 0 1 0}$ \\
Photon exchange & $-\mathbf{0 . 0 0 0 8}$ & $\mathbf{0 . 0 0 0 3}$ \\
Centre-of-mass energy & $-\mathbf{0 . 0 0 3 4}$ & $\mathbf{- 0 . 0 0 1 3}$ \\
\hline Total & $+\mathbf{0 . 0 0 8 5} \pm \mathbf{0 . 0 0 0 7}$ & $\mathbf{+ 0 . 0 0 5 8 \pm \mathbf { 0 . 0 0 1 0 }}$ \\
\hline \hline
\end{tabular}

Table 7: Corrections which need to be applied to the bottom and charm asymmetries $[20,22,24]$ for the measurement on the $\mathrm{Z}^{0}$ resonance. The shifts shown are added to the measured asymmetries to obtain the corrected values. The uncertainties in the QCD corrections are much larger than those on the other corrections.

This result is compared to the LEP average [23] of $\sin ^{2} \theta_{\mathrm{W}}^{\text {eff,lept }}=0.23160 \pm 0.00049$. A recent OPAL measurement of the same quantity can be found in [25], based on the $\tau$ polarisation. Assuming lepton universality that result was $\sin ^{2} \theta_{\mathrm{W}}^{\text {lept }, \mathrm{eff}}=0.2334 \pm 0.0012$. Good agreement is found between the measurements.

According to eq. 2 and eq. 3 the ratio of the effective weak coupling constants can be derived if the lepton coupling parameter, $\mathcal{A}_{l}$, is known. In [25] this parameter is found to be $\mathcal{A}_{l}=0.132 \pm 0.020$. Using the measurement of the charm asymmetry, the charm quark coupling parameter, $\mathcal{A}_{c}$, is evaluated to be

$$
\mathcal{A}_{c}=0.78 \pm 0.17 \text {. }
$$

This can be compared to the result determined from the LEP and SLD average [23] $\mathcal{A}_{c}=$ $0.662 \pm 0.042$. Good agreement is again seen between the measurements. Further, the ratio of the electroweak couplings can be calculated from the charm quark coupling parameter, $\mathcal{A}_{c}$, following eq. 3. The ratio of the effective vector- to axial-vector coupling constants of the charm quark to the $Z^{0}$ is found to be

$$
\frac{g_{\mathrm{V}}^{\mathrm{c}}}{g_{\mathrm{A}}^{\mathrm{c}}}=0.48 \pm 0.17
$$

Using the relations $g_{\mathrm{R}}=g_{\mathrm{V}}-g_{\mathrm{A}}$ and $g_{\mathrm{L}}=g_{\mathrm{V}}+g_{\mathrm{A}}$, the ratio of the left to the right handed effective weak coupling constants are found to be $g_{L}^{\mathrm{c}} / g_{R}^{\mathrm{c}}=-2.9 \pm 1.0$.

\section{$9 \quad$ Results and Summary}

The forward-backward asymmetries of the processes $\mathrm{e}^{+} \mathrm{e}^{-} \rightarrow c \bar{c}$ and $\mathrm{e}^{+} \mathrm{e}^{-} \rightarrow b \bar{b}$ have been measured using $32634 \pm 200 \mathrm{D}^{*+}, \mathrm{D}^{0}$ and $\mathrm{D}^{+}$candidate events containing 28261 signal events on the peak of the $\mathrm{Z}^{0}$ resonance, 1762 events below and 2611 above the peak of the resonance. The results of the fits with both the $b$ and the $c$ asymmetries as free parameters are

$$
\begin{array}{lll}
A_{\mathrm{FB}}^{\mathrm{c}}=0.039 \pm 0.051 \pm 0.009 & A_{\mathrm{FB}}^{\mathrm{b}}=-0.086 \pm 0.108 \pm 0.029 & \left\langle E_{c m}\right\rangle=89.45 \mathrm{GeV} \\
A_{\mathrm{FB}}^{\mathrm{c}}=0.063 \pm 0.012 \pm 0.006 & A_{\mathrm{FB}}^{\mathrm{b}}=0.094 \pm 0.027 \pm 0.022 & \left\langle E_{c m}\right\rangle=91.22 \mathrm{GeV} \\
A_{\mathrm{FB}}^{\mathrm{c}}=0.158 \pm 0.041 \pm 0.011 & A_{\mathrm{FB}}^{\mathrm{b}}=-0.021 \pm 0.090 \pm 0.026 & \left\langle E_{c m}\right\rangle=93.00 \mathrm{GeV}
\end{array}
$$


with a statistical correlation coefficient between the bottom and charm asymmetry of -0.281 on-peak ( -0.272 and -0.273 for the low and high energy off-peak points, respectively). These results are in good agreement with the OPAL measurements using high momentum leptons [14] and other measurements at LEP [26]. From these measurements a value of the weak mixing angle and the ratio of the neutral current weak coupling constants have been extracted.

The results are consistent with the standard model predictions of $A_{\mathrm{FB}}^{\mathrm{c}}=\mathbf{0 . 0 6 2}$ and $A_{\mathrm{FB}}^{\mathrm{b}}=$ 0.095, obtained from the program ZFITTER [20] at a centre-of-mass energy of $91.22 \mathrm{GeV}$, assuming a top mass of $180 \mathrm{GeV}$ and a Higgs boson mass of $300 \mathrm{GeV}$. The observed energy dependence is also consistent with expectation.

\section{Acknowledgements}

We particularly wish to thank the SL Division for the efficient operation of the LEP accelerator, for the precise information on the absolute energy, and for their continuing close cooperation with our experimental group. In addition to the support staff at our own institutions we are pleased to acknowledge the Department of Energy, USA, National Science Foundation, USA, Particle Physics and Astronomy Research Council, UK, Natural Sciences and Engineering Research Council, Canada, Israel Ministry of Science, Israel Science Foundation, administered by the Israel Academy of Science and Humanities, Minerva Gesellschaft, Japanese Ministry of Education, Science and Culture (the Monbusho) and a grant under the Monbusho International Science Research Program, German Israeli Bi-national Science Foundation (GIF), Direction des Sciences de la Matière du Commissariat à l'Energie Atomique, France, Bundesministerium für Bildung, Wissenschaft, Forschung und Technologie, Germany, National Research Council of Canada,

Hungarian Foundation for Scientific Research, OTKA T-016660, and OTKA F-015089. 


\section{References}

[1] For a recent review see e.g. T. Behnke, D.G. Charlton, Phys. Scri. 52 (1995) 133.

[2] OPAL Collaboration, K. Ahmet et al., Nucl. Instr. Meth. A 305 (1991) 275;

P.P. Allport et al., Nucl. Instr. Meth. A 324 (1993) 34;

P.P. Allport et al., Nucl. Instr. Meth. A 346 (1994) 476;

O. Biebel et al., Nucl. Instr. Meth. A 323 (1992) 169;

M. Hauschild et al., Nucl. Instr. Meth. A 314 (1992) 74.

[3] JADE Collaboration, W. Bartel et al., Z. Phys. C 33 (1986) 23;

JADE Collaboration, S. Bethke et al., Phys. Lett B 213 (1988) 235.

[4] OPAL Collaboration, R. Akers et al., Z. Phys. C 65 (1995) 17.

[5] OPAL Collaboration, G. Alexander et al., Z. Phys. C 52 (1991) 175.

[6] T. Sjöstrand, Comp. Phys. Comm. 82 (1994) 74.

[7] OPAL Collaboration, G. Alexander et al., Z. Phys. C 69 (1996) 543.

[8] C. Peterson et al., Phys. Rev. D 27 (1983) 105.

[9] J. Allison et al., Nucl. Instr. Meth. A 317 (1991) 47.

[10] OPAL Collaboration, R. Akers et al., Z. Phys. C 60 (1993) 601.

[11] OPAL Collaboration, R. Akers et al., Z. Phys. C 67 (1995) 27.

[12] OPAL Collaboration, G. Alexander et al., A study of Charm Hadron Production in $\mathrm{Z}^{0} \rightarrow \mathrm{c} \overline{\mathrm{c}}$ and $\mathrm{Z}^{0} \rightarrow \mathrm{b} \overline{\mathrm{b}}$ decays at $L E P$, CERN-PPE/96-51, to be published in Z. Phys. $\mathrm{C}$

[13] OPAL Collaboration, G. Alexander et al., Z. Phys. C 70 (1996) 357.

[14] OPAL Collaboration, P.D. Acton et al., Z. Phys. C 58 (1993) 523.

[15] Particle Data Group, Review of Particle Properties, Phys. Rev. D 54 (1996) 1.

[16] OPAL Collaboration, R. Akers et al., Phys. Lett. B 327 (1994) 411.

[17] R. D. Field and R. P. Feynman, Nucl. Phys. B136 (1978) 1.

[18] OPAL Collaboration, R. Akers et al., Z. Phys. C 67 (1995) 365.

[19] The working group on LEP energy: LEP energy calibration in 1993, 1994 and 1995, LEP energy group/96-05, 22 July 1996.

[20] Calculated using the ZFITTER program described in:

D. Bardin et al., CERN-TH 6443/92 (May 1992); Phys. Lett. B255 (1991) 290; Nucl. Phys. B351 (1991) 1; Z. Phys. C44 (1989) 493.

[21] OPAL Collaboration, R. Akers et al., Phys. Lett. B 353 (1995) 595. 
[22] The LEP Collaborations, ALEPH, DELPHI, L3 and OPAL, and the LEP Electroweak working group, CERN-PPE/96-017, to be published in Nuclear Instruments and Methods.

[23] The LEP Collaborations, ALEPH, DELPHI, L3 and OPAL, and the LEP Electroweak Working Group, CERN-PPE/95-172, and references therein. This paper has been prepared by the LEP collaboration for presentation at major conferences, and contains some preliminary numbers.

[24] B. Lampe, MPI-Ph/93-74;

A. Djouadi, B. Lampe and P.M. Zerwas, Z. Phys. C67 (1995) 123

[25] OPAL Collaboration, G. Alexander et al., A Precise Measurement of the Tau Polarisation and its Forward-Backward Asymmetry at LEP, CERN-PPE/96-78, to be published in Z. Phys. C

[26] DELPHI Collaboration, P. Abreu et al., Z. Phys. C 66 (1995) 341;

ALEPH Collaboration, D. Buskulic et al., Phys. Lett. B 352 (1995) 479. 


\section{OPAL}
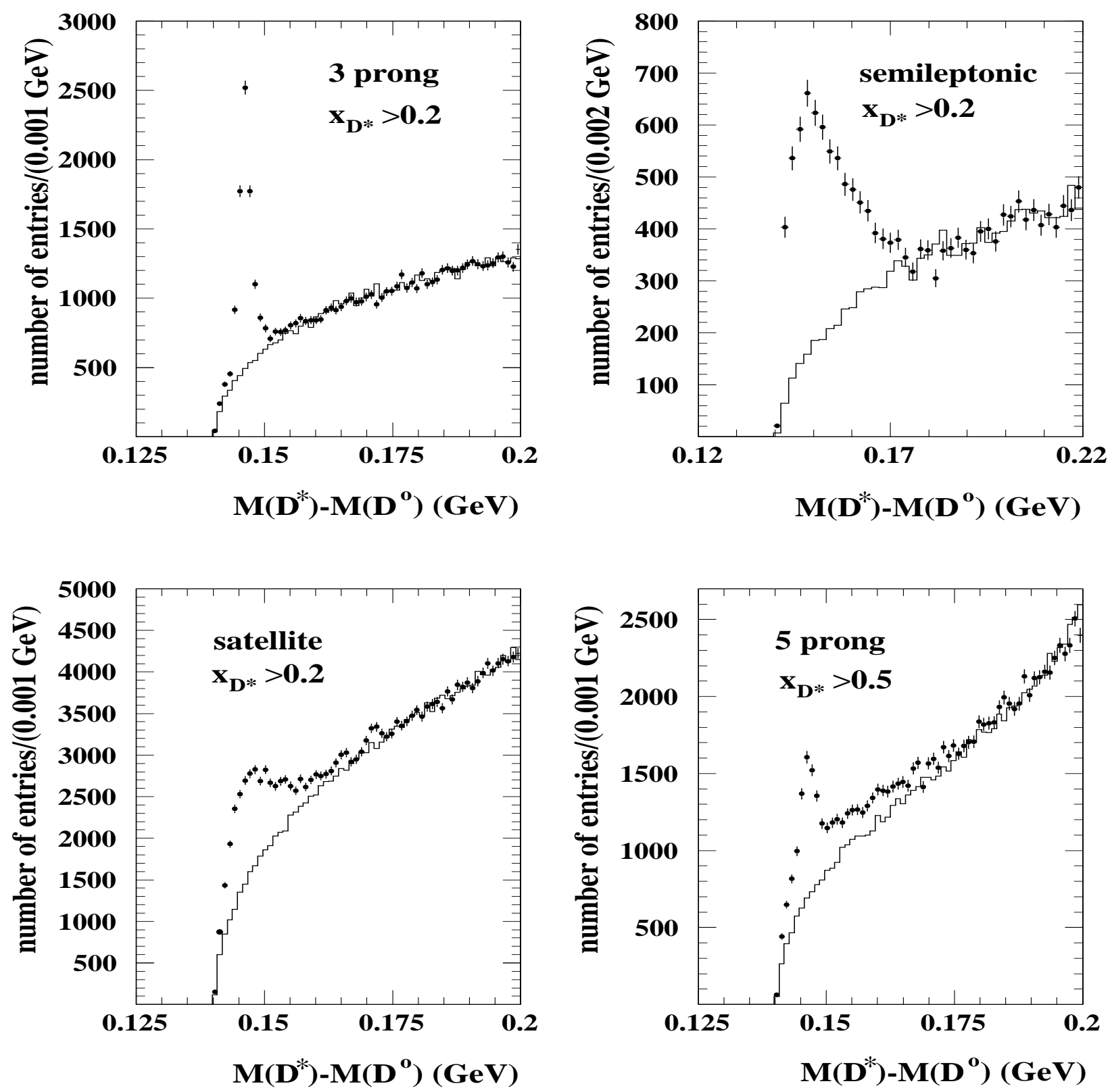

Figure 1: Distributions of the difference $M_{\mathrm{D}^{*+}}-M_{\mathrm{D}^{0}}$ reconstructed in the four different $\mathrm{D}^{*+}$ channels. Both semileptonic decays have been combined into one plot. Superimposed are the background estimator distributions, normalised to the upper sidebands in $\Delta M$. 

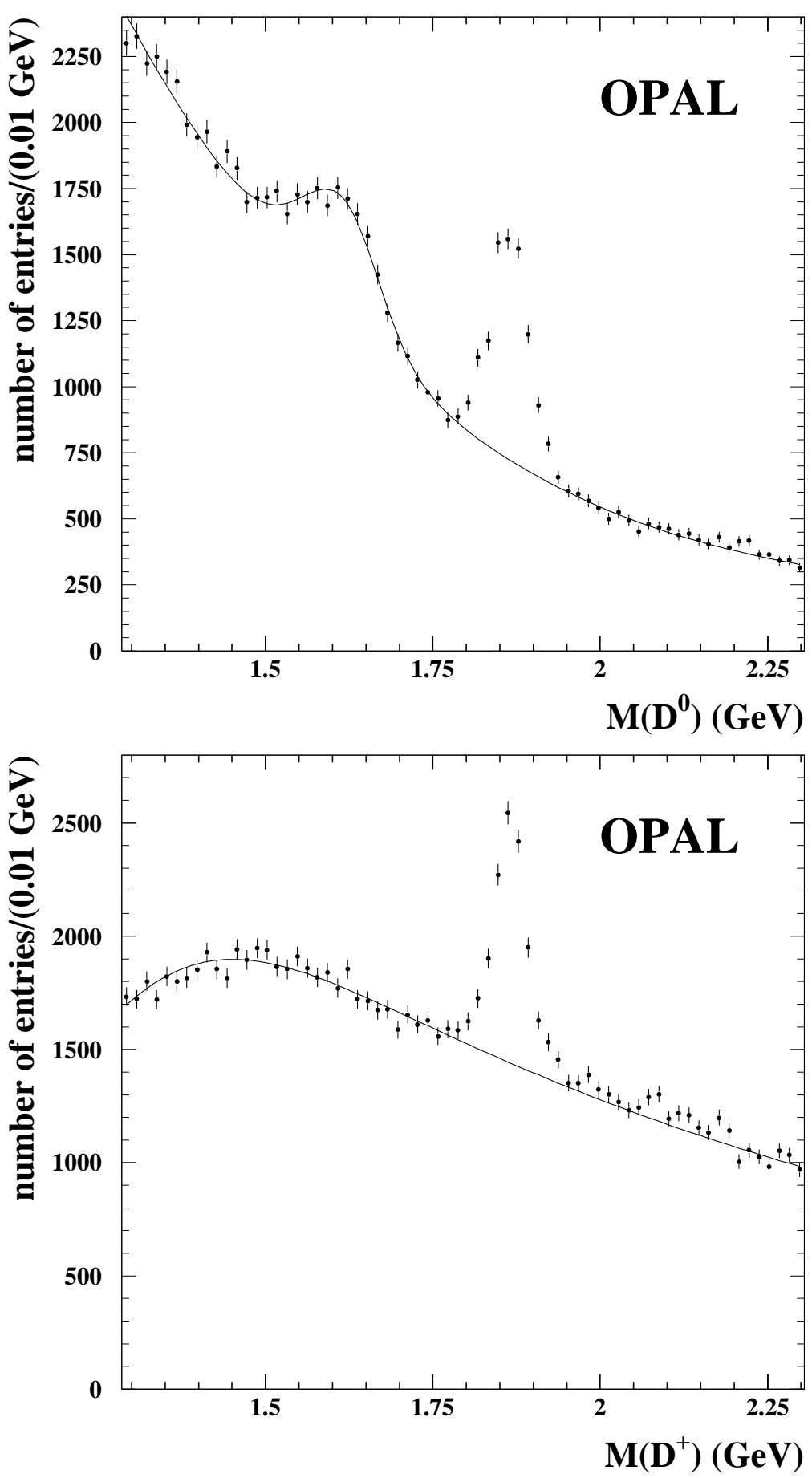

Figure 2: Invariant mass spectra for the $\mathrm{D}^{0}$ and the $\mathrm{D}^{+}$channels. Superimposed is the result of the fit used to determine the number of $\mathrm{D}$ mesons in each sample. 

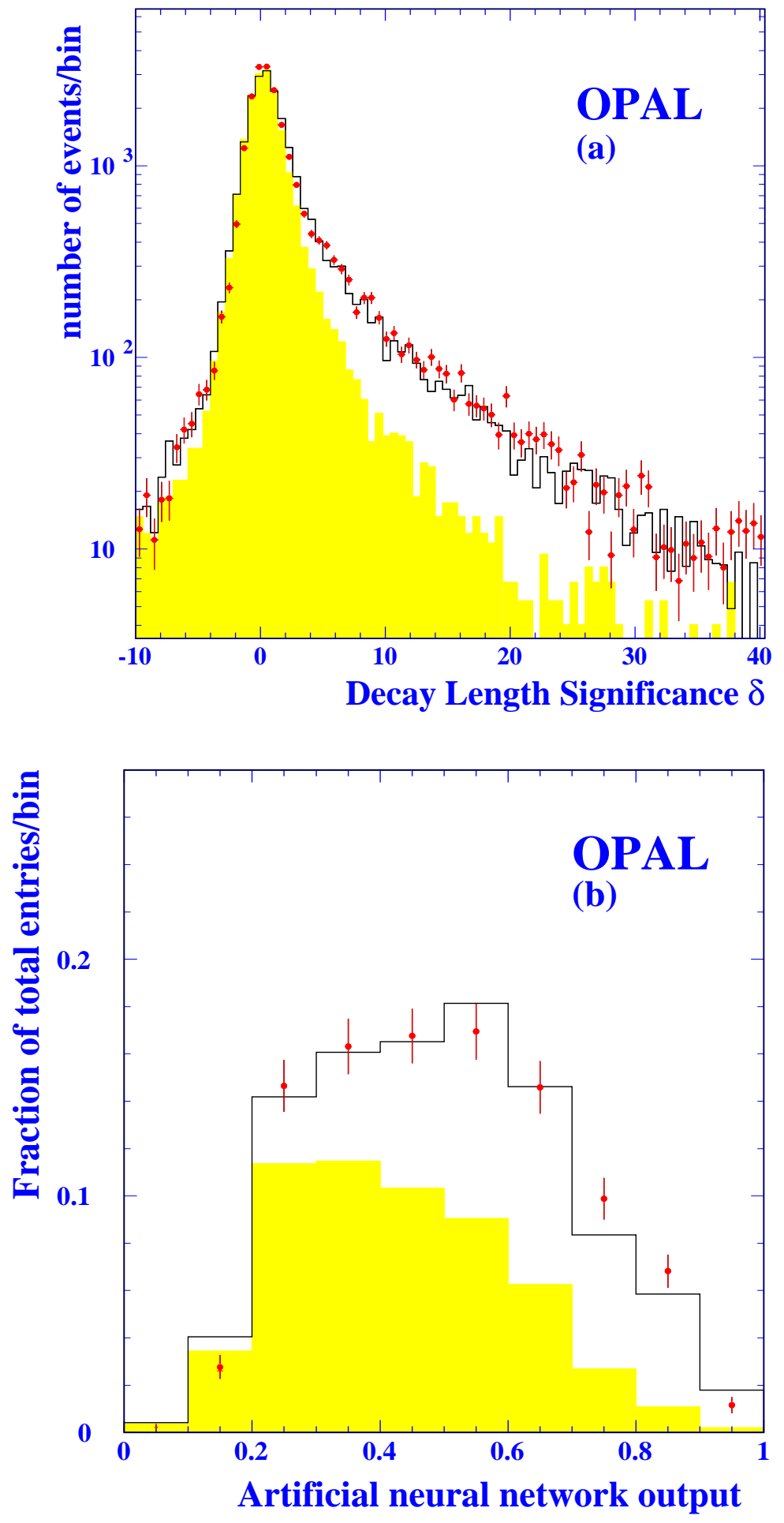

Figure 3: (a) Distribution of the decay length significance in data (points with error bars) and Monte Carlo (solid histogram), as reconstructed in the opposite hemisphere from the $\mathrm{D}^{*+}$ candidate. The shaded histogram represents the distribution for $c \bar{c}$ events, as calculated in the simulation, and normalised to the measured charm fraction in the sample. (b) Normalised distribution of the neural network output for $\mathrm{D}^{*+}$ candidates (points with error bars) and the Monte Carlo distribution for all and $c \bar{c}$ events as in (a). 


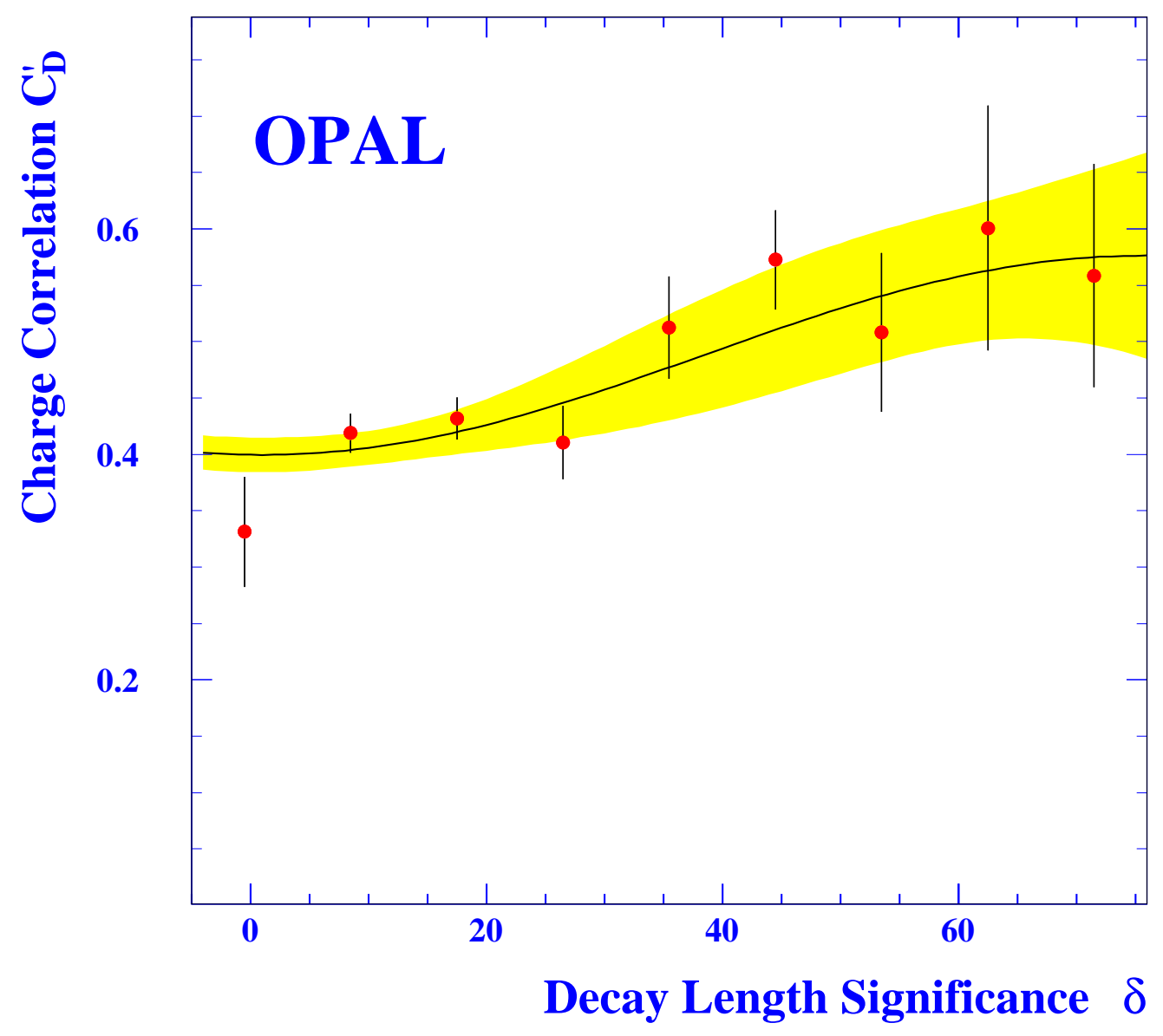

Figure 4: The ratio of unlike sign to all events, after background subtraction, as a function of the decay length significance. Superimposed is the fitted function $C_{d}^{\prime}$ with its error band. 


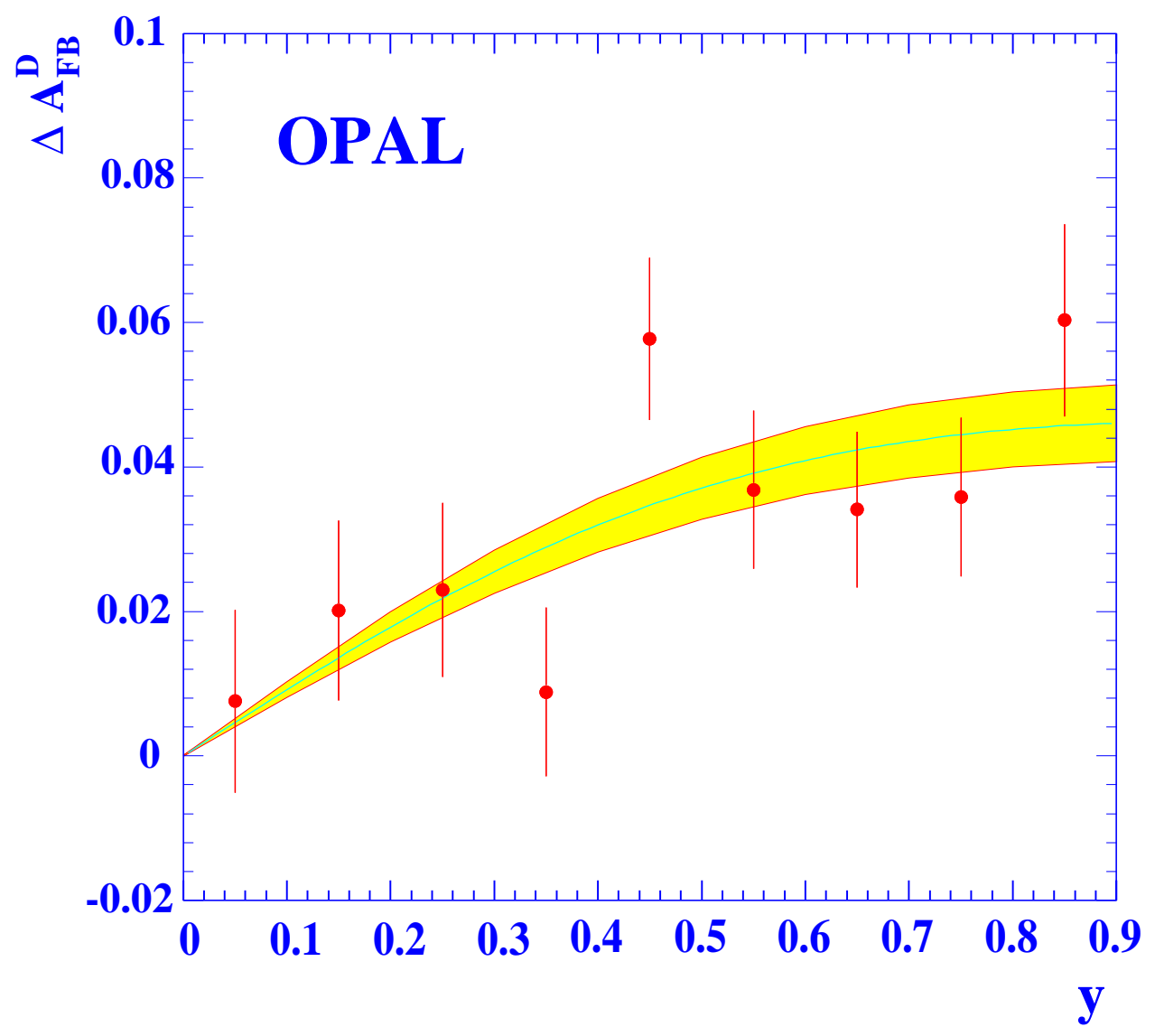

Figure 5: Differential asymmetry as a function of $y=q \cos \theta_{\text {thrust }}$, for all candidates, for on peak energies. Superimposed is the result of the fit. The one standard deviation error is indicated by the shaded band. 

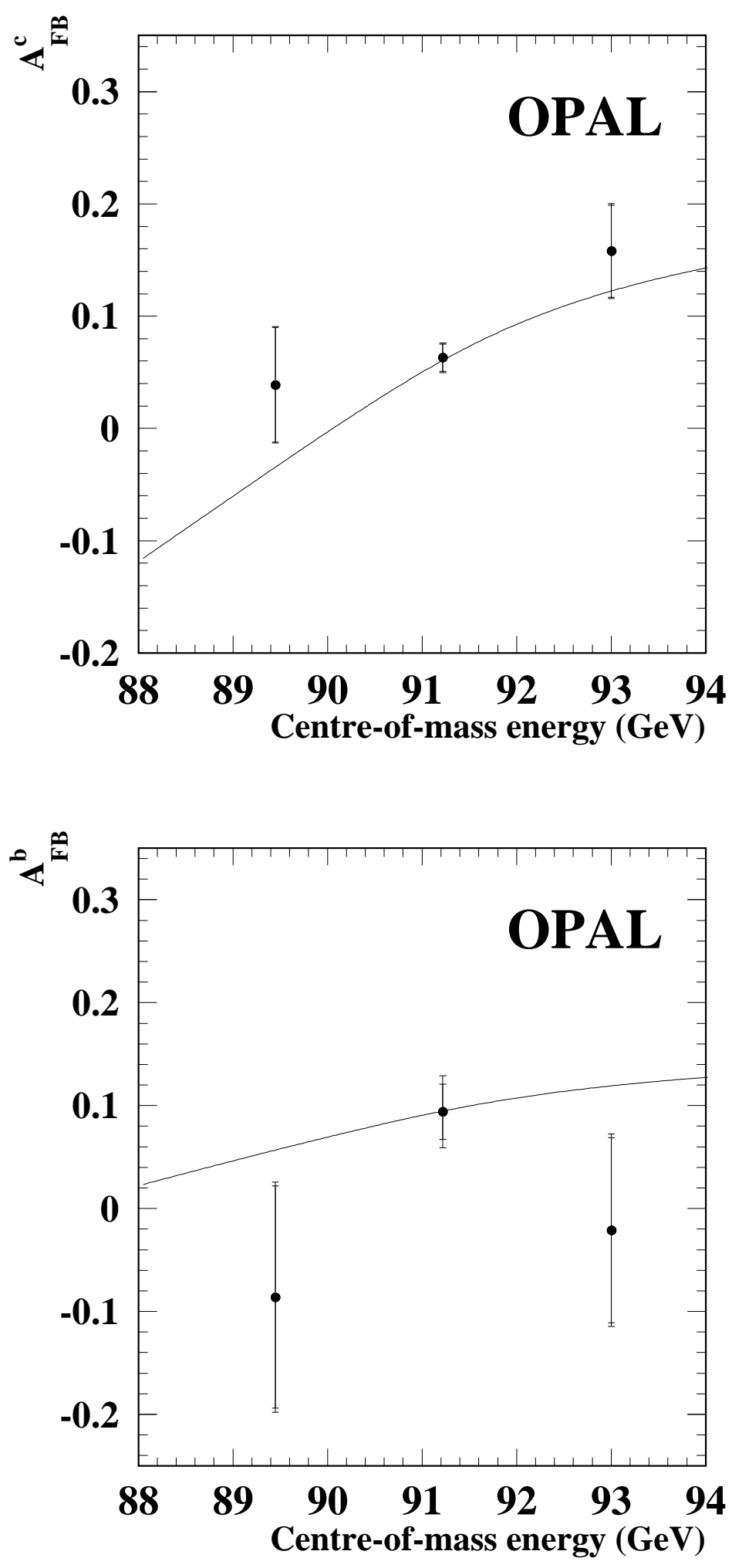

Figure 6: Results for the $\mathrm{b}$ and $\mathrm{c}$ asymmetries at energies on and around the pole of the $\mathrm{Z}^{0}$ resonance. The curve shown is the prediction of the standard model for a Higgs mass of $300 \mathrm{GeV}$ and a top mass of $180 \mathrm{GeV}$. The inner error bars are the statistical errors, the outer ones the total error. 


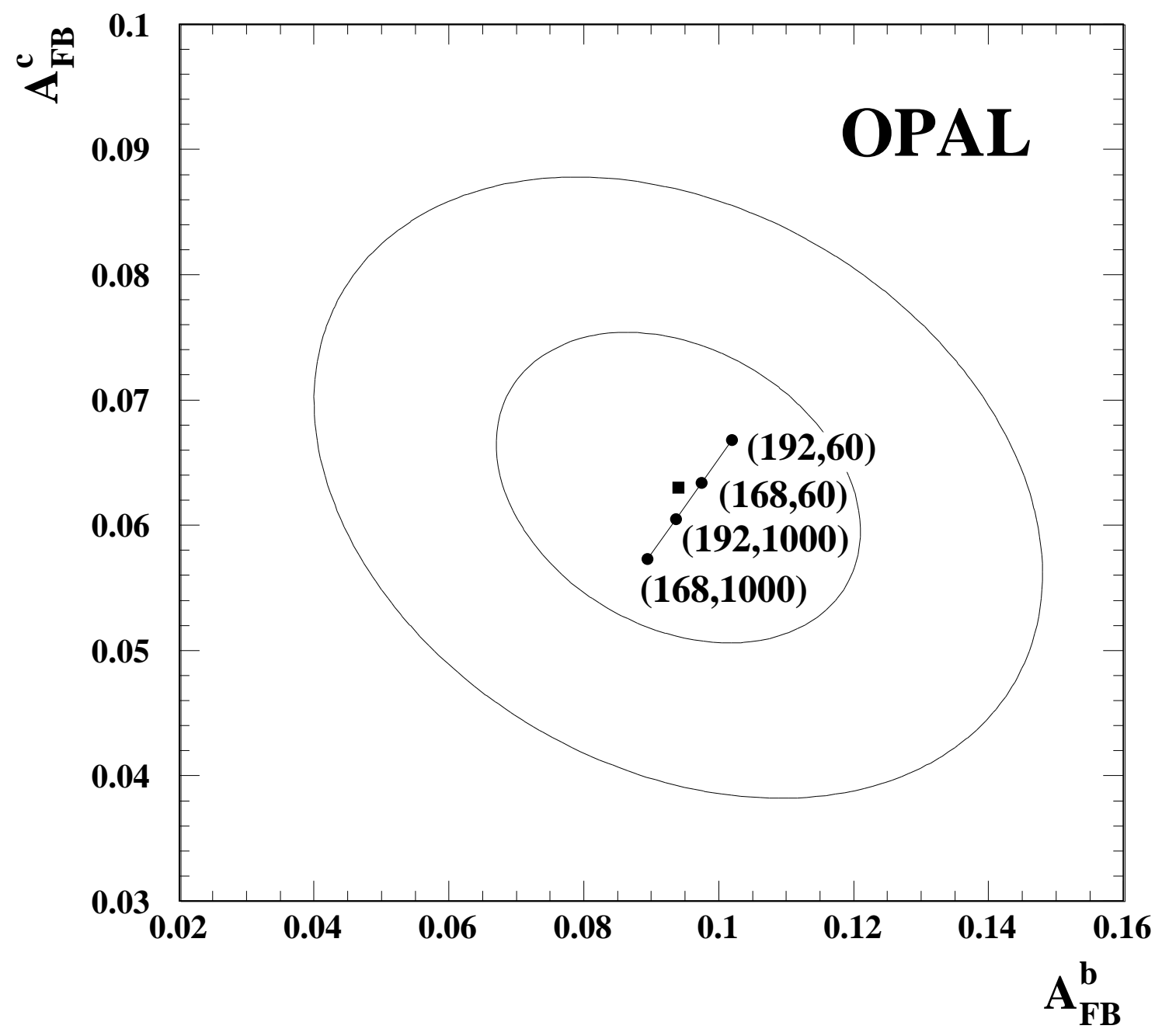

Figure 7: Comparison of the measured $b$ and $c$ asymmetries on the peak of the $Z^{0}$ resonance with the standard model prediction for a range of top and Higgs masses. The top mass is measured [15] to be $(180 \pm 12) \mathrm{GeV}$ and is varied within its errors. The central value is indicated by the square, the inner and outer contours correspond to $39 \%$ and $86 \%$ probability, respectively. The line shown illustrates the standard model predictions for the values $\left(m_{\text {top }}, m_{\text {higgs }}\right)$ shown. 